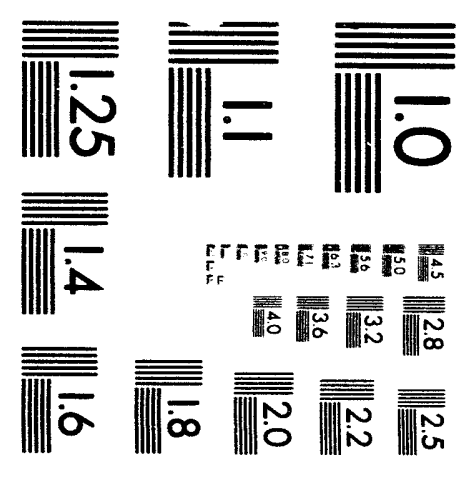



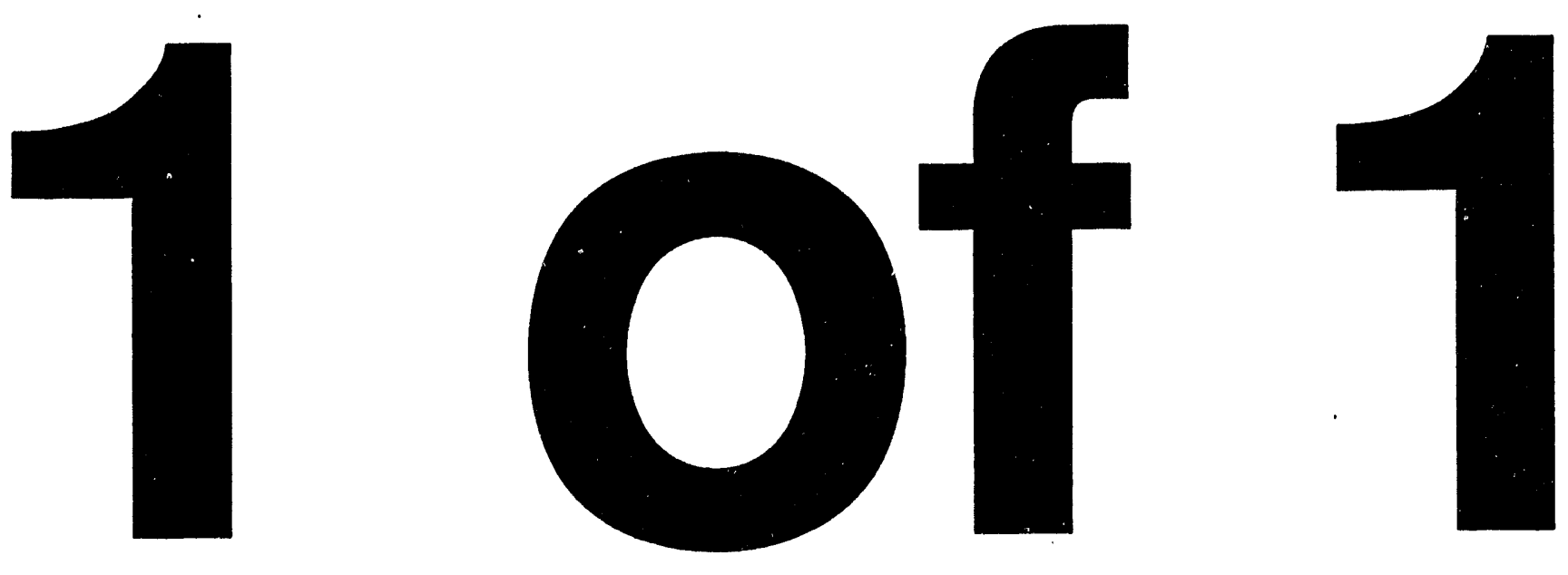


\section{DOE/ET/53088- -T4 \\ INSTITUTE FOR FUSION STUDIES}

Shear Flow Generation from the Interaction of Neoclassical and Drift Wave Transport Processes X.N. Su, P.N. Yushmanov, J.Q. Dong, and W. HorToN Institute for Fusion Studies The University of Texas at Austin Austin, Texas 78712

October 1993

\section{THE UNIVERSITY OF TEXAS}

\section{AUSTIN}




\title{
Shear Flow Generation from the Interaction of Neoclassical and Drift Wave Transport Processes
}

\author{
X.N. Su, P.N. Yushmanov, J.Q. Dong, and W. Horton \\ Institute for Fusion Studies \\ The University of Texas at Austin \\ Austin, Texas 78712
}

\begin{abstract}
Self-consistent shear flow generation from the interaction of neoclassical and drift wave turbulence effects is investigated. The neoclassical poloidal flow damping is shown to compete with the flow generation driven by the divergence of the Reynolds stress. When there is no external driving force except for the free energy released from toroidal shear flow, the turbulent fluctuations occur as a transient pulse which takes the system along an equilibrium path to a relaxed state. External torques, such as parallel neutral beam injection, are needed to maintain significant fluctuation levels. For a system driven by a fixed ion temperature gradient, although linearly the poloidal shear flow generated substantially reduces the growth rate, the simulation results show that a sequence of nonlinear pulses occurs that eventually build the fluctuations up to a level that is not significantly affected by the poloidal flow. In this new, highly nonlinear state the transport is intermittent occurring through a sequence of pulses of duration $100 L_{n} / c_{\rho}$ for typical parameters.
\end{abstract}

\section{DISCLAIMER}

\footnotetext{
This report was prepared as an account of work sponsored by an agency of the United States Government. Neither the United States Government nor any agency thereof, nor any of their employees, makes any warranty, express or implied, or assumes any legal liability or responsibility for the accuracy, completeness, or usefulness of any information, apparatus, product, or process disclosed, or represents that its use would not infringe privately owned rights. Reference herein to any specific commercial product, process, or service by trade name, trademark, manufacturer, or otherwise does not necessarily constitute or imply its endorsement, recommendation, or favoring by the United States Government or any agency thereof. The views and opinions of authors expressed herein do not necessarily state or reflect those of the United States Government or any agency thereof.
} 


\section{Introduction}

Recently, the effects of background shear flow on turbulent transport have been subject to intensive studies. ${ }^{1-7}$ These studies are motivated by recent tokamak experimental observations showing significant levels of radially sheared toroidal flow in neutral-beam heated discharge and a sharp increase in the poloidal shear flow in the edge region of tokamaks which occurs simultaneously with the decrease of the fluctuation level. It is believed that the generation of background poloidal shear flow may be responsible for the decrease in the fluctuation level at the plasma edge and for the improvement of plasma confinement, or the L-H transition.

Generally, it is known that a perpendicular shear flow has a strong stabilizing effect, ${ }^{8-11}$ by introducing a radial asymmetry in the eigenfunction which causes modes with different radial wavenumbers to couple and a parallel shear flow can drive instability by releasing free energy. ${ }^{8-11}$ In many earlier works the effects of background perpendicular and parallel shear flow on turbulence have been studied either separately or with fixed background flow. For purely toroidal flows, Waelbroeck et al. ${ }^{11}$ have shown that the perpendicular component of shear flow can overcome the destabilizing effect of the parallel component for large flow shear. However recent studies ${ }^{\mathbf{3} 4}$ suggest that the background poloidal and toroidal flow will be modified through the Reynolds stress when interaction or coupling between turbulent fluctuation and background mean flow becomes strong. Therefore the studies of the combined effect of toroidal and poloidal shear in self-consistently evolving background flow are important to understand the development of fluctuations and plasma transport.

Neoclassical theory has shown that plasma flow in nonuniform toroidal magnetic field will experience poloidal flow damping due to the magnetic pumping effect, ${ }^{12-17}$ or the socalled neoclassical effect. The effect arises due to the fact that when the plasma rotates from the weak to strong toroidal magnetic field, the cross-section of the flux tube becomes 
small because of the flux preservation. Since the collision frequency is finite, the rotation heats the plasma and leads to an irreversible transformation of the kinetic energy of rotation into thermal energy. In a turbulent plasma, therefore, the neoclassical effect competes with the effect of turbulent fluctuation which tends to drive background mean plasma flow and significantly affects the generation and development of mean plasma shear flow.

In this work we focus on the shear flow generation with the interaction of drift wave turbulence effect and the neoclassical effect. We study the two turbulence models, one is parallel flow shear $u_{\|}^{\prime}$ driven instability model and the other is the $\eta_{i}$ instability model. The $u_{\|}^{\prime}$ driven mode, as we know, drives the instability by releasing free energy stored in sheared parallel velocity profile. As the modes grow, the perpendicular shear flow will be driven through the divergence of the Reynolds stress. This shear flow then suppresses the turbulence. In the meanwhile the magnetic pumping effect causes the damping of the mean poloidal flow. From the point of view of energy, the free energy released from toroidal shear flow first goes to drive turbulence, then part of energy goes to the poloidal flow through Reynolds stress. The poloidal flow energy is subject to loss from the plasma heating due to neoclassical viscosity force (or magnetic pumping). Therefore in a closed system, that is, a system without external driving forces, the plasma flow which starts in an unstable state always relaxes to a stable state along an equilibrium path. During the transition the fluctuations grow up driven by the free energy in the sheared flow and then slowly decays (as a power law in time) as a new stable state is approached. To maintain significant fluctuation levels, it is necessary to have external driving forces. The two kinds of external forces corresponding to two different driving mechanisms are studied in this wo:k. One is parallel neutral beam injection which drives the background parallel shear flow: The parallel shear flow then drives the plasma fluctuations. The other is the ion temperature gradient driving force which directly drives the fluctuations. The grown-up modes then drive background poloidal and toroidal flow through the forces arising from the turbulent transport of plasma 
momentum via the Reynolds stresses. The saturation can be achieved when the external forces are balanced by neoclassical effect and the viscosity force acting on the fluctuations.

This work is organized as follows. Section II gives an introduction to the neoclassical flow. The basic derivation of self-consistent background flow evolution equations with neoclassical effect and drift-acoustic wave turbulence effect, and the equations of the mode evolution are also given in this section. In Sec. III, linear stability analysis is briefly presented. In Sec. IV, we show and discuss the results of the 2-D numerical simulations. Summary and conclusions are given in Sec. V.

\section{Neoclassical Flows}

First order plasma flow $\mathbf{v}$ that lies within a magnetic surface is determined in the toroidally symmetric confinement systems by two magnetic surface functions

$$
u_{\|}=\frac{\langle\mathbf{v} \cdot \mathbf{B}\rangle}{B_{0}} \quad u_{\perp}=\frac{B_{0}}{2 \pi R_{0} B_{P}}\left\langle\mathbf{v} \cdot\left(\frac{\boldsymbol{\nabla} \psi \times \mathbf{B}}{B^{2}}\right)\right\rangle
$$

and can be written in the following form ${ }^{12}$

$$
\mathbf{v}=\frac{\mathbf{B}}{B_{0}}\left(u_{\|}-\frac{B_{T}}{B_{P}} u_{\perp}\right)+u_{\perp} \frac{B_{0}}{R_{0} B_{P}} R^{2} \nabla \phi
$$

where $\mathbf{B}=R_{0} B_{T} \boldsymbol{\nabla} \phi+(1 / 2 \pi)(\boldsymbol{\nabla} \phi \times \nabla \psi), B_{P}$ and $B_{T}$ are poloidal and toroidal magnetic field components, respectively, $B_{0}=\left\langle B^{2}\right\rangle^{1 / 2}=\left\langle B_{P}^{2}+B_{T}^{2}\right\rangle^{1 / 2}, \psi$ is poloidal magnetic flux, $\phi$, $\theta$ are toroidal and poloidal angles and angle brackets means averaging over magnetic surface

$$
\langle A\rangle=\frac{\int \frac{A d \theta}{\mathbf{B} \cdot \nabla \theta}}{\int \frac{d \theta}{\mathbf{B} \cdot \nabla \theta}} .
$$

In Eq. (2) the first term represents incompressible plasma flow along magnetic field lines and the second one describes the rigid body rotation of a magnetic surface. Rigid body rotation (the second term in Eq. (2)) does not contribute to the lowest order viscosity, therefore standard neoclassical viscosity force can depend only on the combination $u_{\|}-\left(B_{T} / B_{P}\right) u_{\perp}$. 
The connection of the perpendicular flow velocity $u_{\perp}$ defined in Eq. (1) with radial electric field $E_{\mathrm{r}}$ is provided by the radial force balance equation which gives

$$
u_{\perp}=-\left(2 \pi B_{0} c / R_{0} B_{p}\right)\left\langle R^{2} B_{p}^{2} / B^{2}\right\rangle\left(\frac{d \Phi}{d \psi}+\frac{1}{e n} \frac{d P}{d \psi}\right)
$$

where $E_{r}$ is determined by electrostatic potential $\Phi(\psi)$ and $P(\psi)$ is the ion pressure. The velocity $u_{\perp}$ is defined such that positive $E_{r}$ gives positive $u_{\perp}$ which is rotation in the ion diamagnetic direction.

Plasma rotation equations in toroidally symmetric systems describe the time evolution of surface functions $u_{\|}$and $u_{\perp}$. We can obtain these equations from momentum balance equation following standard procedure described, for example, in Hassam and Kulsrud, ${ }^{13}$ Hirshman, ${ }^{14}$ and Ware and Wiley ${ }^{15}$

$$
\begin{gathered}
n m\left(1+2 \hat{q}^{2}\right) \frac{\partial u_{\perp}}{\partial t}=-F_{\perp}^{R}-F_{\sim}^{R}-F^{n c}-F_{\perp}^{a}, \\
n m \frac{\partial u_{\|}}{\partial t}=\frac{B_{P}}{B_{T}} F^{n c}-F_{\|}^{R}-F_{\|}^{a},
\end{gathered}
$$

where the standard toroidal geometry enhancement factor is

$$
2 \hat{q}^{2}=\left(\frac{B_{T}}{B_{P}}\right)^{2}\left(1-\frac{1}{\left\langle R^{2}\right\rangle\left\langle R^{-2}\right\rangle}\right) \approx 2 q^{2},
$$

and the last reduction is for large aspect ratio. In deriving Eqs. (3) and (4) we take the plasma velocity as $v+\tilde{v}$ with the mean velocity $v$ given by Eq. (2) in terms of $u_{\downarrow}$ and $u_{\|}$ and $\tilde{\mathrm{v}}$ to be determined by the fluctuation dynamics. Forces marked by index $R$ represent momentum generation produced by fluctuation-related Reynolds stress

$$
\begin{aligned}
& F_{\|}^{R}=\left\langle n m \frac{\mathbf{B}}{B_{0}} \cdot((\tilde{\mathbf{v}} \cdot \boldsymbol{\nabla}) \tilde{\mathbf{v}})\right\rangle ; \\
& F_{\perp}^{R}=\left\langle n m \frac{B_{0}(\boldsymbol{\nabla} \psi \times \mathbf{B})}{2 \pi R_{0} B_{P} B^{2}} \cdot((\tilde{\mathbf{v}} \cdot \boldsymbol{\nabla}) \tilde{\mathbf{v}})\right\rangle ; \\
& F_{\sim}^{R}=\left\langle n m \frac{B_{T}}{B_{P}}\left(\frac{B_{0}^{2}}{B^{2}}-1\right) \frac{\mathbf{B}}{B_{0}} \cdot((\tilde{\mathbf{v}} \cdot \boldsymbol{\nabla}) \tilde{\mathbf{v}})\right\rangle ;
\end{aligned}
$$

and 


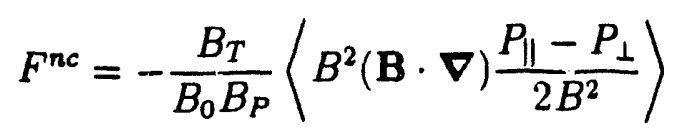

describes neoclassical viscosity force emerging when plasma is pushed along the nonuniform magnetic field (the magnetic pumping effect) and terms marked by index a represent flow damping due to the breakdown of toroidal symmetry of the system that is always present in real devices

$$
\begin{aligned}
& F_{\perp}^{a}=\left\langle\left[\frac{B_{0} \nabla \psi \times \mathbf{B}}{2 \pi R_{0} B_{P} B^{2}}+\frac{B_{T}}{B_{P}}\left(\frac{B_{0}^{2}}{B^{2}}-1\right) \frac{\mathbf{B}}{B_{0}}\right] \cdot \mathbf{F}^{a}\right\rangle, \\
& F_{\|}^{a}=\left\langle\frac{\mathbf{B}}{B_{0}} \cdot \mathbf{F}^{a}\right\rangle .
\end{aligned}
$$

The force $\mathbf{F}^{a}$ can describe the effect of toroidal ripples or charge exchange collisions of ions with neutral gas.

According to Ref. 12 neoclassical viscosity is proportional to poloidal rotation velocity and can be written in the form

$$
F^{n c}=-3 \mu^{n c} \frac{B_{T}}{B_{0}^{2} B_{P}}\left\langle\left(\frac{(\mathbf{B} \cdot \nabla) B}{B}\right)^{2}\right\rangle\left(u_{\|}-\frac{B_{T}}{B_{P}} u_{\perp}-k_{\nu_{\star}} \frac{c}{e B_{P}} \frac{d T_{i}}{d r}\right),
$$

where $\mu^{n c}$ is the neoclassical viscosity coefficient, that for the standard neoclassical assumptions of low rotation velocity $u \ll c_{s}$, scales as ${ }^{12,16}$

$$
\mu^{n c} \approx R_{0} q \frac{n m V_{t h} \nu_{*}}{1+\nu_{*}} \frac{1}{1+\epsilon^{3 / 2} \nu_{*}},
$$

where $\nu_{*}=q R_{0} \nu / V_{t h} \epsilon^{3 / 2}, \nu$ is ion collision frequency, $\epsilon=r / R_{0}$ is inverse aspect ratio, $V_{t h}$ is thermal velocity of the plasma component and the coefficient $k_{\nu}$ describes the relative effect of parallel heat flux on the longitudinal viscosity, ${ }^{17}$ which has following values:

$$
\begin{array}{lll}
k_{\nu_{*}}=1.17 & \text { for } & \nu_{*} \ll 1 \\
k_{\nu_{*}}=-0.5 & \text { for } & 1 \ll \nu_{*} \ll \epsilon^{-3 / 2} \\
k_{\nu_{*}}=-2.1 & \text { for } & \nu_{*} \gg \epsilon^{-3 / 2} .
\end{array}
$$


Here we do not want to specify any particular model of a toroidally asymmetric process that produces the nonambipolar forces $F_{\perp}^{a}$ and $F_{\|}^{a}$. We use somewhat heuristic forms of the forces for the description of the process:

$$
\begin{aligned}
& F_{\perp}^{a}=n m \nu_{\perp}^{a}\left(u_{\perp}-u_{\perp}^{a}\right), \\
& F_{\|}^{a}=n m \nu_{\|}^{a}\left(u_{\|}-u_{\|}^{a}\right) .
\end{aligned}
$$

The forms of the nonambipolar forces given in Eqs. (14) and (15) may describe the process, such as charge exchange with neutral beams (or beam injection) that can be derived from hydrodynamical equations.

Substituting Eqs. (11)-(15) into Eqs. (3) and (4), we obtain the following equation for mean plasma rotation in toroidally symmetric system

$$
\begin{gathered}
\left(1+2 q^{2}\right) \frac{\partial u_{\perp}}{\partial t}=-\nu^{n c}\left(u_{\perp}-S_{0} u_{\|}+u^{n c}\right)-\nu_{\perp}^{a}\left(u_{\perp}-u_{\perp}^{a}\right)-\frac{\partial}{\partial x}\left\langle\tilde{v}_{x} \tilde{v}_{\perp}\right\rangle-2 q\left\langle\cos \theta \frac{\partial}{\partial x}\left(\tilde{v}_{x} \tilde{v}_{\|}\right)\right\rangle \\
\frac{\partial u_{\|}}{\partial t}=-\nu^{n c} S_{0}\left(S_{0} u_{\|}-u_{\perp}-u^{n c}\right)-\nu_{\|}^{a}\left(u_{\|}-u_{\|}^{a}\right)-\frac{\partial}{\partial x}\left\langle\tilde{v}_{x} \tilde{v}_{\|}\right\rangle,
\end{gathered}
$$

where

$$
\begin{aligned}
& \nu^{n c}=\frac{3 \mu^{n c}}{n m} \frac{B_{T}^{2}}{B_{P}^{2} B_{0}^{2}}\left\langle\left(\frac{(\mathbf{B} \cdot \nabla) B}{B}\right)^{2}\right\rangle \approx \frac{3}{2} \frac{B_{T}^{2}}{B_{P}^{2}} \frac{\sqrt{\epsilon}}{1+\nu_{*}}\left(\frac{\nu L_{n}}{c_{s}}\right), \\
& S_{0}=\frac{B_{P}}{B_{T}} \text { and } u^{n c}=k_{\nu_{*}} \frac{c}{e B_{T}} \frac{d T_{i}}{d r}\left(\frac{L_{n}}{\rho_{s} c_{s}}\right) .
\end{aligned}
$$

In writing Eqs. (16) and (17) we reduce the cross-field gradient by $\partial / \partial \psi=\left(2 \pi R B_{p}\right)^{-1} \partial / \partial x$ and use the high aspect ratio approximation $(\epsilon \ll 1)$.

The first terms on the right-hand side of Eqs. (16) and (17) give the neoclassical damping of the poloidal rotation velocity $u_{\theta}=u_{\perp}-S_{0} u_{\|}$to the equilibrium value $u_{0}=-u^{\text {nc }}$ given in Eq. (19). This equilibrium poloidal velocity determined by the ion temperature gradient is in the ion diamagnetic direction for $\nu_{*}>1$ when $k_{\nu_{*}}<0$ and in the electron direction 
for $\nu_{*}<1$ when $k_{\nu_{*}}>0$. The toroidal velocity $u_{\phi}=u_{\|}+S_{0} u_{\perp}$ is not damped by the neoclassical term $\nu^{\text {nc }}$ due to the toroidal symmetry assumed in calculating the $F^{n c}$ force in Eq. (11).

The perpendicular and parallel mean flows are thus determined by the competition between the neoclassical transport coefficients $\nu^{n c}, \nu_{\perp}^{a}, \nu_{\|}^{a}$ and the turbulent (Reynolds) flow tensors given by $\pi_{x \perp}=\left\langle\tilde{v}_{x} \tilde{v}_{\perp}\right\rangle$ and $\pi_{x \|}=\left\langle\tilde{v}_{x} \tilde{v}_{\|}\right\rangle$.

The dynamical equations for the fluctuation fields $\tilde{\varphi}$ and $\tilde{v}_{\|}$in the local slab geometry are taken as ${ }^{6,9,18}$

$$
\begin{aligned}
& \left(1-\nabla_{\perp}^{2}\right) \frac{\partial \tilde{\varphi}}{\partial t}+v_{d} \frac{\partial \tilde{\varphi}}{\partial y}+\left(\frac{\partial^{2} u_{\perp}}{\partial x^{2}}+\left(u_{\perp}+S_{0} u_{\|}\right)\left(1-\nabla_{\perp}^{2}\right)\right) \frac{\partial \tilde{\varphi}}{\partial y}-\left[\tilde{\varphi} \quad, \nabla_{\perp}^{2} \tilde{\varphi}\right]=-\nabla_{\|} \tilde{v}_{\|}-\mu_{\perp} \nabla_{\perp}^{4} \tilde{\varphi} \\
& \frac{\partial \tilde{v}_{\|}}{\partial t}+\left(u_{\perp}+S_{0} u_{\|}\right) \frac{\partial \tilde{v}_{\|}}{\partial y}-\frac{\partial u_{\|}}{\partial x} \frac{\partial \tilde{\varphi}}{\partial y}+\left[\tilde{\varphi} \quad, \tilde{v}_{\|}\right]=-\nabla_{\| \tilde{\varphi}}+\mu_{\perp} \nabla_{\perp}^{2} \tilde{v}_{\|}
\end{aligned}
$$

where

$$
\begin{aligned}
& \nabla_{\perp}=\hat{\mathbf{x}} \frac{\partial}{\partial x}+\hat{\mathbf{y}} \frac{\partial}{\partial y}, \\
& \nabla_{\|}=\frac{\partial}{\partial z}+\left(L_{n} / \rho_{s}\right) S_{0} \frac{\partial}{\partial y} \\
& {[f, g]=\frac{\partial f}{\partial x} \frac{\partial g}{\partial y}-\frac{\partial f}{\partial y} \frac{\partial g}{\partial x}}
\end{aligned}
$$

and $u_{\perp}=u_{\perp}(x, t)$ and $u_{\|}=u_{\|}(x, t)$ are the background perpendicular rotation and parallel flow velocity field given by Eqs. (16) and (17), respectively.

In Eqs. (20) and (21), $\mu_{\perp}$ is the dissipation coefficient from collision ion viscosity and $S_{0}=B_{y} / B_{0}\left(\simeq B_{P} / B_{T}\right)$ is magnetic tilting angle.

The usual drift wave scaling are used in the non-dimensional variables in above equations:

$$
\begin{aligned}
& x^{\prime}, y^{\prime}=x / \rho_{s}, y / \rho_{s}, \quad z^{\prime}=z / L_{n} ; \\
& t^{\prime}=c_{s} t / L_{n} ;
\end{aligned}
$$




$$
\varphi^{\prime}=\left(\frac{L_{n}}{\rho_{\mathrm{s}}}\right)\left(\frac{e \Phi}{T_{\mathrm{e}}}\right)
$$

and all the velocities, $u_{\perp}, u_{\|}, \tilde{v}_{\|}$and $\tilde{\mathbf{v}}_{\perp}\left(=\left(\rho_{s} c_{s} / L_{n}\right) \mathbf{b} \times \boldsymbol{\nabla}_{\perp} \tilde{\varphi}\right)$, are in units of the electron diamagnetic drift velocity $v_{d e}=\rho_{s} c_{s} / L_{n}$.

Equations (20) and (21) together with Eqs. (16) and (17) are the basic model equations used in our simulations with the results given in Sec. IV. In the following sections we use the dimensionless variables and omit the primes. These nonlinear coupled equations for the neoclassical evolution of the mean flows and the dynamics of the fluctuations for:n a new hybrid neoclassical-drift wave turbulence model for the toroidal plasma.

The total energy $E$ is dissipated only by the small dissipation from perpendicular viscosity $\mu_{\perp}$ and the neoclassical drags due to collisional relaxations of the anisotropics driven by the rotation. The fluctuations transfer the background flow energies $\left(E_{b g 1}\right)$ and $\left(E_{b g 2}\right)$ through the two (Reynolds) tensors. So we find it useful to define and study the four energy components obtained by dividing the poloidal or $\mathbf{E} \times \mathbf{B}$ flow and the toroidal or parallel flow into their background $(b g)$ mean flow components and into their fluctuating $(f l)$ kinetic components. The definition of these four components are as follows:

$$
\begin{aligned}
& E_{b g, 1}=\frac{1}{2} \int_{V}\left(1+2 q^{2}\right) u_{\perp}^{2} \frac{d V}{L_{x} L_{y}}, \\
& E_{f l, 1}=\frac{1}{2} \int_{V}\left(\tilde{\varphi}^{2}+\left(\nabla_{\perp} \tilde{\varphi}\right)^{2}\right) \frac{d V}{L_{x} L_{y}}, \\
& E_{b g, 2}=\frac{1}{2} \int_{V} \dot{u}_{\|}^{2} \frac{d V}{L_{x} L_{y}}, \\
& E_{f l, 2}=\frac{1}{2} \int_{V} \tilde{v}_{\|}^{2} \frac{d V}{L_{x} L_{y}} .
\end{aligned}
$$

The Reynolds tensors are defined by

$$
\pi_{x \perp}=\left\langle\tilde{v}_{x} \tilde{v}_{\perp}\right\rangle=-\int \frac{d y}{L_{y}} \frac{\partial \tilde{\varphi}}{\partial x} \frac{\partial \tilde{\varphi}}{\partial y}=\operatorname{Im} \sum_{k_{y}} k_{y} \tilde{\varphi}_{k_{y}}^{*} \frac{\partial \tilde{\varphi}_{k_{y}}}{\partial x}
$$




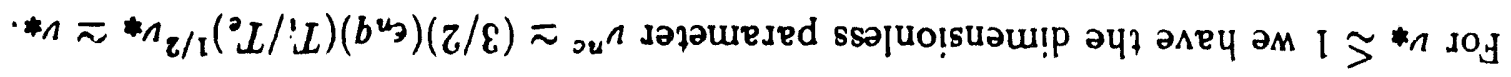

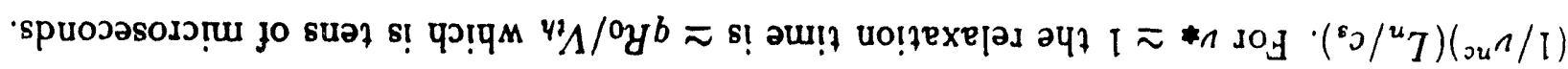

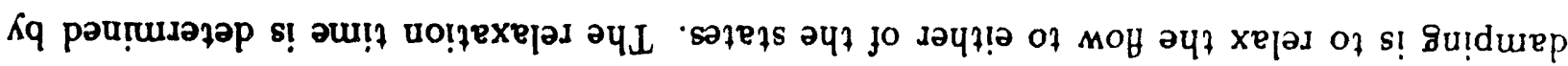

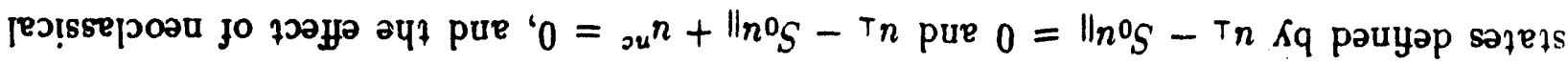
un!

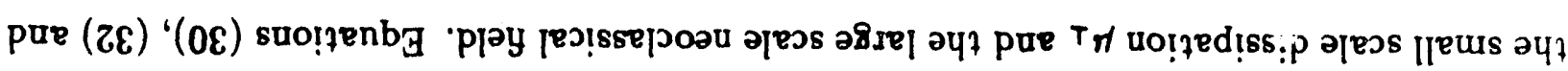

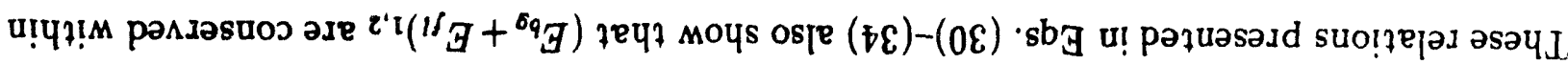

$$
\begin{aligned}
& \cdot{ }^{x} \frac{1}{x p}\left\|x \Perp \frac{x \varrho}{\|_{n}} \int-=\frac{{ }^{x} T}{x p} \frac{x \varrho}{\| x \Perp \varrho}\right\|_{n} \int \\
& \text { ' } \frac{{ }^{x} T}{x p} T^{x} \frac{x \varrho}{T_{n} \varrho} \int-=\frac{{ }^{x} T}{x p} \frac{x \varrho}{T^{x} \Perp \varrho} T_{n} \int
\end{aligned}
$$

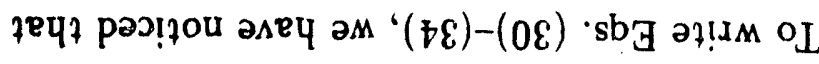

$(\nabla \mathcal{E})$

$$
\begin{aligned}
& \cdot \frac{{ }^{x} T}{x p}\left(\left\|_{v} n-\right\|_{n}\right)^{\|_{n}} \int_{0}^{\Lambda} \|_{n} n-\frac{{ }^{x} T}{x p}\left(T_{v}^{T} n-T_{n}\right)^{\top} n \int{ }_{v}^{T} n- \\
& \frac{{ }^{x} T}{x p}\left[\left({ }_{o u} n+\|_{n} 0 S-T n\right)\left(\|_{n} 0 S-T n\right)\right] \int_{o u} n-
\end{aligned}
$$

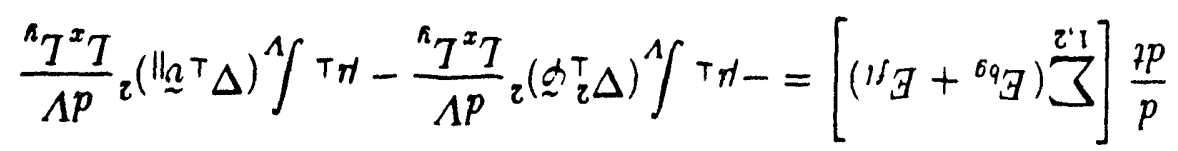

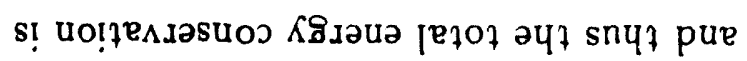

$\varepsilon)$

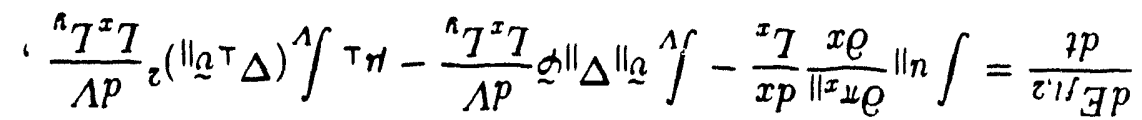

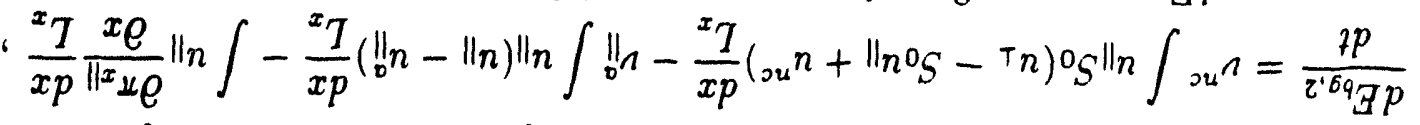

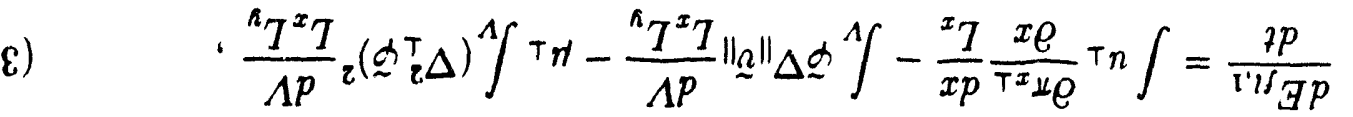

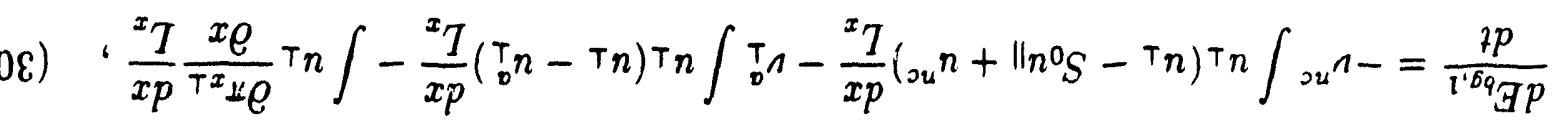

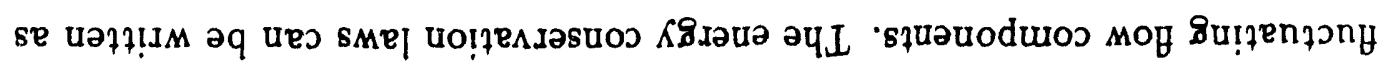

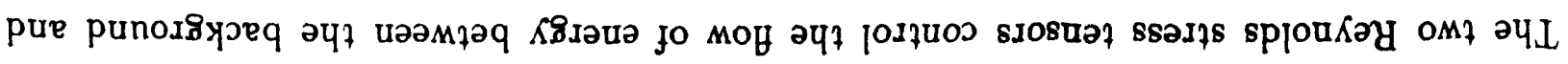

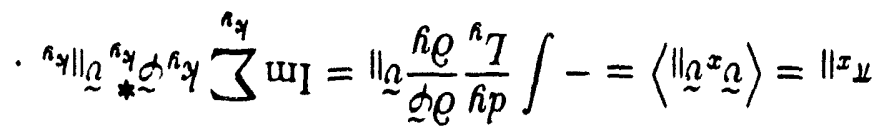




\section{Linear Instability}

We now examine the linear stability of the parallel shear flow driven mode in the magnetic shearless system based on the model equations (20) and (21). Considering the linear modes have the wave form of $\tilde{\varphi}=\tilde{\varphi}_{0} \exp \left[i\left(k_{y} y-\omega t\right)\right]+c . c$. , we can derive the eigenmode equations by linearizing Eqs. (20) and (21) as

$$
\frac{\partial^{2} \tilde{\varphi}}{\partial x^{2}}+\left[-\left(1+k_{y}^{2}\right)+\frac{\omega_{y}+k_{y} u_{\perp}^{\prime \prime}(x)}{\omega-k_{y} u_{\perp}(x)-k_{\|} u_{\|}(x)}-\frac{k_{\|}\left(k_{y} u_{\|\|}^{\prime}(x)-k_{\|}\right)}{\left[\omega-k_{y} u_{\perp}(x)-k_{\|} u_{\|}(x)\right]^{2}}\right] \tilde{\varphi}=0,
$$

where $\omega_{*}=k_{y} v_{d}=L_{n} \omega_{*_{e}} / c_{s}$ is dimensionless diamagnetic rotation. In the quasilinear evolution the flow profiles $u_{\perp}(x, t), u_{\|}(x, t)$ take on complicated structures. For profiles with weak $u_{\perp}^{\prime \prime}$ we may expand the mean perpendicular and parallel velocity as $u_{\perp}(x) \simeq u_{0 \perp}(0)+$ $u_{\perp}^{\prime} x$ and $u_{\|}(x) \simeq u_{0 \| \mid}(0)+u_{\|}^{\prime} x$ and transform to the moving frame so that $u_{0 \perp}(0)=u_{0 \|}(0)=0$ in the moving frame. The laboratory frame frequencies must be Doppler shifted by $k_{y} u_{0 \perp}(0)+$ $k_{\|} u_{0 \|}(0)$ from those obtained in the following stability analysis. Equation (36) is then a Whittaker equation ${ }^{11}$ with no converging mode in $-\infty \leq x \leq \infty$ space due to the fact that $\left(1+k_{y}^{2}\right)>0$. However, if the expansions for $u_{\perp}(x)$ and $u_{\|}(x)$ are assumed to be valid only in a finite domain of $x$ space, then the solutions which converge in the finite domain of $x$ space are relevant and unstable under the conditions given here.

Now we first consider the mode which is well localized such that $\left.\left|k_{y} u_{\perp}^{\prime}+k_{\|} u_{\mu}^{\prime}\right|\right)|| x|\ll| \omega \mid$. We can therefore obtain the lowest order eigenmode equation from Eq. (36)

$$
\frac{\partial^{2} \tilde{\varphi}}{\partial x^{2}}+\left(A+B x-C x^{2}\right) \tilde{\varphi}=0
$$

where

$$
\begin{aligned}
& A=-\left(1+k_{y}^{2}\right)+\frac{\omega_{*}}{\omega}-\frac{k_{\|}\left(k_{y} u_{\|}^{\prime}-k_{\|}\right)}{\omega^{2}} \\
& B=\frac{\left(k_{y} u_{\perp}^{\prime}+k_{\|} u_{\|}^{\prime}\right)}{\omega^{2}}\left[\omega_{*}-\frac{2 k_{\|}\left(k_{y} u_{\|}^{\prime}-k_{\|}\right)}{\omega}\right]
\end{aligned}
$$




$$
C=\frac{\left(k_{y} u_{\perp}^{\prime}+k_{\|} u_{\|}^{\prime}\right)^{2}}{\omega^{3}}\left[\frac{3 k_{\|}\left(k_{y} u_{\|}^{\prime}-k_{\|}\right)}{\omega}-\omega_{*}\right] .
$$

The lowest eigenmode solution of Eq. (37) is given by

$$
\tilde{\varphi}(x)=\exp \left[-\frac{\sqrt{C}}{2}\left(x-\frac{B}{2 C}\right)^{2}\right] \text {. }
$$

For $\omega * \omega<k_{\|}\left(k_{y} u_{\|}^{\prime}-k_{\|}\right)$, the eigenvalue can be determined by

$$
\omega=\frac{\omega_{*} \pm \sqrt{\omega_{*}^{2}-4\left(1+k_{y}^{2}\right)\left[(2 / 3) k_{\|}\left(k_{y} u_{\|}^{\prime}-k_{\|}\right)-\sqrt{3 k_{\|}\left(k_{y} u_{\|}^{\prime}-k_{\|}\right)}\left(k_{y} u_{\perp}^{\prime}+k_{\|} u_{\|}^{\prime}\right)\right]}}{2\left(1+k_{y}^{2}\right)}
$$

The marginal instability can be derived from Eq. (40)

$$
\omega_{*}^{2}-4\left(1+k_{y}^{2}\right)\left[(2 / 3) k_{\|}\left(k_{y} u_{\|}^{\prime}-k_{\|}\right)-\sqrt{3 k_{\|}\left(k_{y} u_{\|}^{\prime}-k_{\|}\right)}\left(k_{y} u_{\perp}^{\prime}+k_{\|} u_{\|}^{\prime}\right)\right]=0 \text {. }
$$

When the dimensionless diamagnetic rotation $\omega_{*}=L_{n} \omega_{*_{e}} / c_{0}$ is small, compared with the flow shears, we obtain

$$
\left(k_{y} u_{\perp}^{\prime}+k_{\|} u_{\|}^{\prime}\right)^{2} \simeq \frac{4}{27} k_{\|}\left(k_{y} u_{\|}^{\prime}-k_{\|}\right)
$$

Equation (42) describes the balance of poloidal shear flow stabilization with the parallel shear flow destabilization. For $k_{\|}=1 / q R$ the first unstable $k_{y}$ is $k_{y}=\left(u_{\|}^{\prime 2}-4 / 27\right) /\left(q R u_{\|}^{\prime}\left(4 / 27-u_{\perp}^{\prime}\right)\right.$ and the fastest growing mode for large $\left|u_{\|}^{\prime}\right|$ is $k_{y}=2 / q R u_{\|}^{\prime}$ with $\gamma_{\max }=2 u_{\|}^{\prime} / \sqrt{\left(q R u_{\|}^{\prime}\right)^{2}+2 q R u_{\|}^{\prime}}$.

In a general case Eq. (36) has to be solved numerically to get eigenvalues $\boldsymbol{\omega}$ and eigenfunctions $\tilde{\mathcal{P}}(x)$. In Figs. $1 \mathrm{a}$ and $1 \mathrm{~b}$ the normalized growth rate contours are shown in $u_{\perp}^{\prime}=\left(L_{n} / c_{s}\right) d V_{E} / d x$ and $u_{\|}^{\prime}=\left(L_{n} / c_{s}\right) d V_{\|} / d x$ space for magnetic shearless and with magnetic shear $\left(k_{\|}=k_{y} x / L\right.$, and $\left.s=L_{n} / L_{0}\right)$ configurations, respectively. The mode with maximum growth rate is chosen among the modes found and the growth rate is not sensitive to the boundary of the region. For the case with magnetic shear (Tig. 1b) the contours with higher growth rate are shown to avoid high $u_{\perp}^{\prime}$ values which makes the stability problem complicated and it will be discussed in a separate work. The interaction of $u_{\|}^{\prime}$ and $\eta_{i}$ destabilization is studied elsewhere ${ }^{9}$ and not considered here. In the presence of $\eta_{i}$ and small $u_{\perp}^{\prime}$ the stability analysis is given in Hamaguchi and Horton (1992). ${ }^{6}$ 


\section{Results of Numerical Simulations}

In this section we report and discuss the results of the nonlinear numerical simulations. We numerically solve Eqs. (16)-(21) using procedures described in Ref. 19. One can study the model in two cases, local and global mode cases. In the local mode case, the magnetic field in the $y$ direction, $B_{y}$, is considered a small finite constant, so that the magnetic parameter $S_{0}=B_{y} / B_{0}=$ const. For the global mode case, $B_{y}$ is a function of $x$, so that $S(x)=B_{y}(x) / B_{0}$ depends on $x$, which gives a $2 \mathrm{D}$ model for the effect of magnetic shear. In the case of a sheared magnetic field, it is important to distinguish between single helicity simulation/theory and full 3D multihelicity simulation/theory as analyzed by Beklemishev and Horton. ${ }^{20} \mathrm{~A}$ recent study ${ }^{21}$ indicates that it is physically more relevant to treat $B_{y} / B_{0}$ as a constant in the two-dimensional case, while in three-dimensional case it is necessary to treat $B_{y} / B_{0}$ as a function of $x$ since there are multiple rational surfaces with overlapping wave functions. In this work we only consider the two-dimensional case, and therefore in all of our simulation cases, we consider $S_{0}$ to be a constant.

In this work, the simulation box is taken to be $L_{x}=L_{y}=20 \pi \rho_{s}$, which is to represent the region of strong shear flow. We usually set initial background poloidal rotation $u_{\perp}(x, t=0)=$ $u_{\perp 0} \sin \left(k_{1} x\right)$ and parallel shear flow $u_{\|}(x, t=0)=u_{\| 0} \sin \left(k_{1} x\right)$, where $k_{1}=2 \pi / L_{x}$, which means in physical units a variation of $u_{\|}$by $u_{\mid p_{0}} c_{s}\left(\rho_{s} / L_{n}\right)$ over the scale of $62 \rho_{0}$ (typically 6 $\mathrm{cm})$. The initial fluctuations of $\tilde{\varphi}$ and $\tilde{v}_{\|}$are usually set $\left.\tilde{\varphi}=\tilde{v}_{\|}=0.1 \exp \left[-\left(x^{2}+y^{2}\right) / r_{0}^{2}\right)\right]$ or, alternatively, $0.1 x \exp \left[-\left(x^{2}+y^{2}\right) / r_{0}^{2}\right]$ with $r_{0}=6\left[\rho_{s}\right]$. The neoclassical flow velocity is $u^{n c}=u_{0}^{n c} \sin \left(k_{1} x\right)$. The set of Eqs. (16)-(21) is solved in an $85 \times 85 k_{x} k_{y}$ space with Runge-Kutta-Verner fifth-order and sixth-order method for advancing in time.

We have tested the accuracy of the nonlinear evolution by studying energy conservation in both the two-field code with $\varphi-v_{\|}$dynamics and the three-field code with $\varphi-v_{\|}-\delta p$ dynamics. Since there is transfer of energy between the fluctuations energies $E_{f l, 1}, E_{f l, 2}$ and $E_{f l, 3}$ and 
between the fluctuation and the background kir tic energ: $\left.: u_{\perp}^{2}\right\rangle$ and $\left\langle u_{\|}^{2}\right\rangle$ the conservation of the total energy requires many transfer terms to exact slance. We find that the codes conserve the total energy well. In one test with all dissipation terms set zero and a large amplitude initial perturbation we find that over the time interval $t c_{s} / L_{n}=[0,500]$, the total energy decreases by

$$
E_{T}(0)-E_{T}(500)=13.06273-13.06239=3.4 \times 10^{-4}
$$

for a relative change of $(3.4 / 13) \times 10^{-4} \simeq 3 \times 10^{-5}$. In this test the background kinetic energy initial' ts at zero, increa ': to approximately unity and settles down to 'tuate around 0.4. The variations in time, se three fluctuation energy crmponents are ider of unity.

In the first three cases of simulation, we study the parallel velocity shear driven mode with the self-consistent background flow evolution with and without the neoclassical damping effect from $\nu^{n c}$. As shown in Ref. 9 with fixed background flow, the two field model equations (20) and (21) include linearly unstable modes driven by free energy released from parallel shear flow. However the background evolution with time is important because when the modes grow up, the fluctuation fields become large and the turbulent fluctuations significantly affect the profile of the background flow throl the divergence of the Reynolds momentum transport tensors $\left\langle\tilde{v}_{x} \tilde{v}_{\perp}\right\rangle$ and $\left\langle\tilde{v}_{x} \tilde{v}_{\|}\right\rangle$as shown in Eqs. (16) and (17). In the fourth case of the simulations, we study the shear flow generation in the ion temperature gradient driven or so-called $\eta_{i}\left(=d \ell n T_{i} / d \ell n n\right)$ mode case in the presence of the background neoclassical effects. 


\section{Case 1: Conversion of Toroidal Flow to Poloidal Flow Through Turbulence}

In the first case $1 \mathrm{~A}$, we set $\nu^{\text {nc }}=0$ and $\nu_{\perp}^{a}=\nu_{\|}^{a}=0$, that is, there is no neoclassical flow, $S_{0}=B_{P} / B_{T}=0.2$ and the perpendicular viscosity $\mu_{\perp}=0.01$ in the evolution of fluctuating fields only, $\mu_{\perp}=0$ in the evolution of background flows. This case may be considered as the limit of a sufficiently high ion temperature plasma that the ion-ion collisional effects are negligible on the time scales of interest.

For the time interval $t=0-300\left(L_{n} / c_{s}\right)$, we keep the background flow fixed, that is, $u_{\perp}=u_{\perp}(x, t)=u_{\perp}(x, t=0)=0$ and $u_{\|}=u_{\|}(x, t)=u_{\|}(x, t=0)=6 \sin \left(k_{1} x\right)$ do not evolve in time throughout this time period $[0,300]$. The modes are driven by $u_{\|}^{\prime}=\partial u_{\|} / \partial x$. A saturated state of vortex turbulence is obtained at about $t=160\left(L_{n} / c_{s}\right)$ to $t=300\left(L_{n} / c_{s}\right)$ as shown in Figs. $2 \mathrm{a}, 3 \mathrm{a}$ and $4 \mathrm{a}$ with rms fluctuation levels of $\tilde{u}_{\perp}=0.3\left(v_{d e}\right)$ and $\tilde{u}_{\|}=0.55\left(v_{d e}\right)$. At $t=300\left(L_{n} / c_{s}\right)$ we turn on the background flow dynamical equations (16) and (17) (with $\left.\nu^{n c}=\nu_{\perp}^{a}=\nu_{\|}^{a}=0\right)$, that is, we allow $u_{\perp}$ and $u_{\|}$to evolve with time. The background poloidal energy $\left(E_{b g, 1}\right)$ is shown to immediately increase and the toroidal energy $\left(E_{b g, 2}\right)$ decrease in Figs. $2 b$ and $3 b$ to create a new stage with a mean helical shear flow. The turbulent fluctuations $\tilde{\varphi}$ and $\tilde{v}_{\|}$are shown to be suppressed as the poloidal shear flow increases in Figs. 2 and 3 for $t>300\left(L_{n} / c_{s}\right)$. Figure 4 is contour plots of $\varphi$ at $t=275\left(L_{n} / c_{s}\right)$ and $t=395\left(L_{n} / c_{s}\right)$. Figure $4 \mathrm{~b}$ shows that for $t>300\left(L_{n} / c_{s}\right)$ the sheared poloidal flow generated is sufficient to produce mostly open stream lines which suppresses the turbulence and the turbulent transport shown in Fig. $4 \mathrm{~b}$ where the poloidal flow is still zero. The turbulent momentum transport generates the poloidal shear flow while reducing the parallel shear flow as shown in Figs. 2, 3 and 4.

In the case $1 \mathrm{~B}$ we set all the parameters and initial profiles to the same values as in case $1 \mathrm{~A}$, but let $u_{\perp}(x, t)$ and $u_{\|}(x, t)$ evolve with time from the beginning $(t=0)$. Like the case $1 \mathrm{~A}$, the modes are driven by $u_{\|}^{\prime}$, however, the gradient drive now relaxes in time. A 
saturated state is now obtained at around $t=50\left(L_{n} / c_{s}\right)$ as shown in Fig. 5a. After reaching this maximum at $t \simeq 50\left(L_{n} / c_{s}\right)$ the turbulence now immediately begins to decay in contrast to case $1 \mathrm{~A}$. Figures 5 and 6 are time evolutions of fluctuation $\left(E_{f l}\right)$ and background $\left(E_{b g}\right)$ energies for poloidal and toroidal flows, respectively. Figure 7 shows the time evolution of the mode fluctuations $\tilde{\varphi}\left(k_{x}, k_{y}=1.0\right)$ for a spectrum of $k_{x} \rho_{s} \rightarrow k_{x}$ values. From Figs. 5 and 6 , we can see that when $t<40\left(L_{n} / c_{s}\right)$, the modes experience exponential growth at the growth rate $\gamma \lesssim 0.1 c_{s} / L_{n}$. When the turbulent fluctuations become large, as in the time interval $40<t c_{s} / L_{n}<8 n$. they drive up the poloidal flow in Fig. 5b. While the shear of the poloidal flow is wr $t$ shows no effect of suppressing the growth of the fluctuations. However, when the $\mathrm{pc}$. lal shear flow gets strong, the mode fluctuations are effectively suppressed, and so the signals from the mode fluctuations become small for $t>100\left(L_{n} / c_{s}\right)$ as shown in Fig. 7. We note from Fig. 6b that the toroidal flow energy decreases with time during the whole process. The most significant drop in the toroidal kinetic energy during the period $30<t c_{s} / L_{n}<80$ coincides with the rise of the poloidal kinetic energy during the peak of turbulent pulse. Part of the toroidal flow energy goes to poloidal flow and another part is damped through the viscous dissipation of the fluctuations. In the final state the ratio of the mean poloidal velocity $\left(u_{\perp} \sim 0.44\left(v_{d e}\right)\right)$ to the mean toroidal velocity $\left(u_{\|}=3.5\left(v_{d e}\right)\right)$ is about $1 / 9$.

\section{Case 2: Neutral Beam Driven Toroidal Shear Flow}

In this case we study the effect of nonambipolar flow on background flow. Physically the nonambipolar flow $u_{\|}^{a}$ can arise from neutral beam injection. We set $\nu_{\|}^{a}=0.1$, $S_{0}=0.2, \mu_{\perp}=0.01$ while keeping $\nu^{n c}=0, \nu_{\perp}^{a}=0$, and the externally driven variation in the toroidal profile $u_{\|}^{a}=10 \sin \left(k_{1} x\right)$. The spatially uniform $u_{0 \|}$ part of $u_{\|}^{a}(x)$ is transformed away by going to the appropriate reference frame. From Eq. (15) this means that a nonuniform streaming parallel momentum with the finite value of the injection rate 
$\nu_{\|}^{a}=0.1$ and speed $u_{\|}^{a}$ now is injected into the plasma. The initial profiles for background flows are $u_{\perp}(x, t=0)=0$ and $u_{\|}(x, t=0)=\sin \left(k_{1} x\right)$, and the fluctuation fields are $\tilde{\varphi}(x, y, t=0)=\tilde{v}_{\|}(x, y, t=0)=0.1 \exp \left(-\frac{x^{2}+y^{2}}{r_{0}^{2}}\right)$ with $r_{0}=6\left[\rho_{s}\right]$. In the beginning stage $\left(t=0-100\left[L_{n} / c_{s}\right]\right), u_{\|}^{\prime}$ is too weak to drive the modes unstable. However the toroidal shear flow increases during this time from the injected parallel flow $u_{\|}^{a}$, which gives a driving force for the toroidal shear fie: $u_{\|}(x, t)$. When the neutral beam driven toroidal shear flow gets large, the shear flow drives the unstable modes. The fully developed fluctuation then transfer energy-momentum to the background poloidal flow through $\pi_{x \perp}$. The mean poloidal flow reaches about one half the mean toroidal flow velocity. With the increase of the poloidal shear flow, again the turbulent fluctuations of the modes are suppressed, but due to the continuous driving a well defined turbulent state is reached with $\tilde{u}_{\perp} \simeq 0.24\left(v_{d e}\right) \gtrsim \tilde{u}_{\|}$. The whole process is shown in Figs. 8 and 9 giving the time evolution of the four energy components. A nonlinear regression fitting code is used here to determine the decay power of the turbulent fluctuation energy. The results show that the total fluctuation energy decays to a constant as $1 / t^{m}$, with the decay index $m$ in the range of 1.5 to 2.5 depending on the system parameter. For the kinetic energy in the parallel velocity fluctuations $E_{f l, 2}(t)$ the parameterization is shown in Fig. 10. In Fig. 10, where the dashed line shows the fluctuation energy $E_{f l, 2}$ versus time and solid line is the fitting curve that determines $m \simeq 2.3$ and the asymptotic value of $E_{f l, 2}(\infty) \cong 0.008$ for this case.

\section{Case 3: Effect of Neoclassical Flow and Damping on the Turbulence}

In this case, we let $\nu^{n c}=0.01, \nu_{\perp}^{a}=\nu_{\|}^{a}=0, S_{u}=0.2, \mu_{\perp}=0.01$ and the neoclassical flow profile $u^{\text {nc }}=4 \sin \left(k_{1} x\right)$ and initially $u_{\perp}(x, t=0)=0$ and $u_{\|}(x, t=0)=20 \sin \left(k_{1} x\right)$. With these profiles, therefore the background flow is initially in the neoclassical equilibrium state so that $u_{\perp}-S_{0} u_{\|}+u^{n c}=0$. With evolution of time, the fluctuations first are driven up due to the parallel shear flow, then again when the background poloidal shear flow becomes 
strong enough $\left(E_{b g, 1} \sim 0.7\right.$ or $u_{\perp} \sim 1\left(v_{d e}\right)$ in this case), the fluctuations are suppressed and decay as $1 / t^{m}$ where $m \sim 1$ as shown in Figs. 11 a and $b$. Meanwhile the parallel shear flow becomes weak due to the release of its free energy to the fluctuations and the perpendicular shear flow as shown in Figs. 12a and b. In the final turbulent state the mean flow fluctuation levels are $\tilde{u}_{\perp} \simeq 1\left(v_{d e}\right)$ and $\tilde{u}_{\|} \simeq 0.5\left(v_{d e}\right)$ similar to earlier cases.

Figure 13 shows the evolution of the mean square deviation of the helical flow field from the neoclassical equilibrium, which is monitored by

$$
E_{n c}(t)=\left\langle\left(u_{\perp}-S_{0} u_{\|}+u^{n c}\right)^{2}\right\rangle
$$

with time. In the final state $E_{n c} \lesssim 0.03$ showing that the background flow goes to another equilibrium state determined by the line $u_{\perp}^{f}-S_{0} u_{\|}^{f}+u^{n c} \simeq 0$. The observed evolution is summarized in Fig. 14 where $f$ means the final quasi-steady state. A quantitative example of the stability boundary for particular parameters is the $\gamma=0$ limit of the contours in Fig. 1.

From other simulations not shown here we find that, in fact, we can start with any ressonable initial state for the background flow, that is, any point in parameter space in Fig. 14, and we find that the turbulent fluctuation will take the system along the equilibrium state line $u_{\perp}^{f}-S_{0} u_{\|}^{f}+u^{n c} \simeq 0$ towards the linearly stable region shown in Fig. 14. This shows that the neoclassical damping $\nu^{n c}$ and the turbulent fluctuation always tend to stabilize the system. Therefore, in the systems studied the turbulent fluctuations can be maintained at a significant level only if there is either an exterior accelerating torque such as neutral beam injection as given in Case 2 to supply energy-momentum to drive the background parallel shear flow or an additional force such as the ion temperature gradient $\left(\partial T_{i} / \partial x\right)$, as studied in the next case, to directly drive the unstable modes. The driven fluctuations of the modes can then produce a continuous acceleration force on the background flow through the divergence of the Reynolds stress tensors. 


\section{Case 4: Shear flow generation and Ion Temperature Gradient Driven Fluctua- tions}

In this case, we study shear flow generation in the $\eta_{i}$ driven turbulence. We still use Eqs. (16) and (17) for the evolution of the background flows. For dynamical equations of the fields $\tilde{\varphi}, \tilde{v}_{\|}$and $\tilde{\rho}$, we use Eqs. (1)-(3) in Ref. 22. The equations for $\tilde{\varphi}$ and $\tilde{v}_{\|}$are similar to Eqs. (20) and (21) with a few changes: the toroidal curvature term $2 \epsilon_{n} \partial \varphi / \partial y$ is added to the right-hand side of Eq. (20); the term $\nabla_{\| \tilde{\varphi}}$ at the right-hand side of Eq. (21) is changed to $\nabla_{\|}(\tilde{\varphi}+\tilde{p})$; and a third fluctuation equation or the thermal balance equation for the ion pressure $\tilde{p}$ is added. We set $\eta_{i}=1.5$, toroidicity parameter $\epsilon_{n}=L_{n} / R=0.1, \tau=T_{e} / T_{i}=1$, $S_{0}=0.2, \Gamma=5 / 3$, dimensionless viscosity $\mu_{\perp 1}=\mu_{\perp 2}=0.2$ and dimensionless collisional thermal conductivity $\kappa=0.1$ for the evolution of the fluctuation fields. We do not repeat the Hong-Horton equations here but refer the readers to Ref. 22.

In this simplified $2 \mathrm{D}$ toroidal $\eta_{i}$-mode model without magnetic field the fluctuation levels in the absence of flows gives vortices that merge into a final dipolar vortex filling the simulation box. The field amplitudes are very large.

For the background flow, we set $\nu^{n c}=0.0, u^{n c}=0, \nu_{\perp}^{a}=\nu_{\|}^{a}=0$ and initially set $u_{\perp}(x, t=$ $0)=u_{\|}(x, t=0)=0$. (The cases with $\nu^{n c}=0.0$ and 0.1 are discussed subsequently.) With evolution in time, the modes first are driven by ion temperature gradient, and background perpendicular and parallel flows then are driven through the divergences of the Reynolds

stresses $\left\langle\tilde{v}_{x} \tilde{v}_{\|}\right\rangle$and $\left\langle\tilde{v}_{x} \tilde{v}_{\perp}\right\rangle$. During the time period of $t=0-180\left(L_{n} / c_{s}\right)$, which is the first nonlinear steady state, the linear growth stops at $t \sim 80\left(L_{n} / c_{s}\right)$ and from $t=100-$ $180\left(L_{n} / c_{s}\right)$ there is a well-defined steady state. The increase of the background shear flow significantly reduces the development of the fluctuation level as verified by comparing with the computer experiment without the background flow buildup in the same time period. Figure 15a shows that without the background shear flow, the total fluctuation energy is saturated as early as $t \sim 100\left(L_{n} / c_{s}\right)$ at $E_{f} \simeq 1400$. While with evolving background flow 
in Fig. 15b, the total fluctuation energy growth stops first at $t \simeq 80\left(L_{n} / c_{s}\right)$ with $E_{f} \simeq 35$ and the rms fluctuation level is about 6 times less than that in Fig. 15a in the first nonlinear state.

When the time progresses to $t \sim 600\left(L_{n} / c_{s}\right)$ in the system with the evolving shear flow, the saturation of the total fluctuation energy is observed as shown in Fig. 16a. Figures 16a and $16 \mathrm{~b}$ show that the saturation is characterized by the $180^{\circ}$ out of phase oscillations of the fluctuation energy and background kinetic energy, which is caused by the energy exchanges between the fluctuations and the background through the energy fluxes given in Eq. (35). The total average fluctuation energy level, however, is about the same as that shown in Fig. 15a in which the background flow is fixed at $u_{\perp}=0$ and $u_{\|}=0$. This indicates that the background shear flow can greatly reduce the linear growth rate of the modes and the first nonlinear saturation level, but does not significantly affect the long-time limit saturation level of the average fluctuation in the nonlinear regime. Similar phenomena are also observed in earlier numerical studies for collisional drift wave turbulence and resistive pressure-gradient-driven turbulence by Carreras et al.4,23

The phenomena of nonlinear growth are further confirmed when we study the effects of the neoclassical damping. We made three numerical experiments with $\nu^{\text {nc }}=0,0.01$, and 0.1 . The results show that although the neoclassical damping reduces the development and build-up of the background flow, especially reducing the perpendicular flow velocity, the time-average levels of the total fluctuation energies in the final stage in all three cases remain about the same as that in the case without the background flow shown in Fig. 15a. Figures 17 and 18 show the total fluctuation and background kinetic energies for $\nu^{n c}=0.01$ and 0.1 , respectively. For a clearer comparison, in Figs. $19 \mathrm{a}$ and $\mathrm{b}$ we plot the total fluctuation energies and background kinetic energies for the different $\nu^{\text {nc }}$ values, where the dot, solid and dashed line are for $\nu^{n c}=0.1,0.01$ and 0 , respectively. As we can see from Figs. 19a and $b$, at the final saturation stage although the background kinetic energy levels are quite 
different for the different $\nu^{n c}$, the fluctuation energy levels have no significant difference. The results for the variation of $\nu^{n c}$ are summarized in Table I.

In searching for the mechanism that maintains the fluctuation levels given in Table I at about the same level with different background flow levels shown in Table I, we have considered the role of $u_{\perp}^{\prime \prime}$ and $u_{\|}^{\prime}$ as possible destabilizing mechanisms. To test these possibilities we carried out simulations in a fixed background with large $u_{\perp}^{\prime \prime}$ fields typical of those found in the quasi-steady states between the bursts of growth in Figs. 16-18 and with a fixed $u_{\|}^{\prime}(x)$ field. The results for the fixed profiles show that the fluctuation levels $\sqrt{2 E_{f l}}$ reach to about 8 , then relax to $\sim 1$ due to the suppression from the strong perpendicular flow shear. Thus, the results of this study establish the qualitative importance of the nonlinear evolution of the $u_{\perp}(x, t)$ profile. In terms of renormalized turbulence ideas, the explanation for the behavior is that the evolving profiles of $u_{\perp}(x, t)$ and $u_{\|}(x, t)$ change the effective potential of Eq. (36) (generalized to include $\eta_{i}$ ) to such forms that there is always some new wave trapping region in which a new unstable mode can grow up. These growth phases would account for the twenty-odd growing parts of the turbulent pulses shown in Fig. 16a. Each pulse is determined by the nonlinear saturation of this quasilinear eigenmode which has caused the velocity profiles to change sufficiently to form a new region of wave trapping and growing. This process is repeated over and over giving the series of bursts of turbulence that are out-of-phase with the increase in the high shear flow states shown in Figs. 16-18.

\section{Summary and Conclusions}

In this work, we have studied the control of shear flow generation through the interaction of drift wave turbulence and neoclassical flow with self-consistent background flow profile evolution in parallel velocity shear driven turbulence and ion temperature gradient driven turbulence. Both the perpendicular and parallel background mean flows self-consistently evolve with time and are modified by the plasma turbulence through the Reynolds stress. 
The generation of the background shear flow therefore is a result of the coupling between the turbulent fluctuations and the background mean flow. In a nonuniform toroidal magnetic field, the neoclassical viscosity effect acts as a drag force which transfers the kinetic energy in the poloidal rotation into thermal energy.

We show when the fluctuation is driven by the free energy released from the parallel velocity shear, without external energy supply (such as neutral beam injection), the toroidal plasma always relaxes from an unstable state to a stable state along an equilibrium path which is determined by the neoclassical condition $u_{\perp}-S_{0} u_{\|}+u^{\text {nc }}=0$ from Eqs. (16) and (17). Typically the development of the $u_{\|}^{\prime}$ driven turbulence is the following: parallel shear flow drives the fluctuations by releasing the free energy stored in the sheared toroidal velocity profile. The fully developed fluctuations then push on the background and drive the perpendicular shear flow through the divergence of the Reynolds stress $\left\langle\tilde{v}_{x} \tilde{v}_{\perp}\right\rangle$. Thus if the neoclassical transport coefficient $\nu^{\text {nc }}$ is small, the perpendicular shear flow will grow large and then suppresses the turbulence. If $\nu^{\text {nc }}$ is large, however, the background flow will rapidly relax to an equilibrium state given by $u_{\perp}-S_{0} u_{\|}+u^{n c}=0$. Therefore the magnitude of $u_{\perp}$ depends on that of the neoclassical flow $u^{\text {nc }}(x)$ (where $S_{0}=B_{p} / B_{T}$ ) given in Eq. (11). In the case where $d u^{n c} / d x$ is large, the perpendicular shear can rather abruptly become strong enough to suppress the turbulence. A similar abrupt stabilization of the turbulence is also observed by Sugama and Horton ${ }^{7}$ using resistive-g turbulence. In both cases (large or small $\nu^{n c}$ ), the poloidal flow loses energy to the plasma due to the neoclassical viscosity force defined in Eq. (11), which is also called the magnetic pumping effect. Therefore the system finally goes to a stable state with the turbulence being the mechanism for the evolution.

For an open system in which an external source keeps supplying energy to the system, as in the cases of the neutral beam injection and the ion temperature gradient driven mode, the saturation can be achieved when the external driving forces are balanced by neoclassical damping and the viscous and thermal dissipation. The results of two dimensional simulations 
have shown that the generation of the perpendicular shear flow can effectively reduce the growth rate and slow down the development of the fluctuations. Additional considerations for the shear flow control of turbulent transport including the effect of poloidal asymmetry in the anomalous flux and the role of biased probes is given by Hassam et al. ${ }^{24}$

In the case of a strong ion temperature gradient driven toroidal mode, without magnetic shear, we find that the nonlinear evolution of the poloidal velocity profile keeps allowing a sequence of modes to go unstable that in turn allows an overall nonlinear growth of the spectrum up to the level found in the absence of the poloidal shear flow. Thus, it appears that in the late nonlinear stage, the background shear flow has no significant effect on the saturation level of turbulent fluctuations. In other words, the background shear flow can greatly delay the occurrence of the high level of turbulent fluctuations and the associated transport, but it does not finally eliminate the turbulent transport as previously thought. The nature of the time dependence of the transport is predicted to change from a steady state transport to an intermittent transport state. In the simulation the bursts occur on the time scale of $100\left(L_{n} / c_{s}\right) \sim 100 \mu \mathrm{sec}$. Further study is required to obtain the parametric dependence of the intermittency and the associated effective transport rates. 


\section{Acknowledgments}

The authors acknowledge useful discussions with H.V. Vong and F.L. Waelbroeck. The work was supported by the U.S. Department of Energy contract \#DE-FG05-80ET-53088. 


\section{References}

1. K.C. Shaing and E.C. Crume, Jr., Phys. Rev. Lett. 63, 2369 (1989).

2. J.F. Drake, J.M. Finn, P. Guzdar, V. Shapiro, V. Shevchenko, F. Waelbroeck, A.B. Hassam, C.S. Liu, and R. Sagdeev, Phys. Fluids B 4, 488 (1992).

3. P.H. Diamond and Y.B. Kim, Phys. Fluids B 3, 1626 (1991).

4. B.A. Carreras, V.E. Lynch, L. Garcia, and P.H. Diamond, Phys. Fluids B 5, 1491 (1993).

5. K.C. Shaing, Phys. Fluids B 4, 171 (1992).

6. S. Hamaguchi and W. Horton, Phys. Fluids B 4, 319 (1992).

7. H. Sugama and W. Horton, Shear Flow Generation by Reynolds Stress and Suppression of Resistive $g$ Modes, to be published in Phys. Fluids B, 1993.

8. J.Q. Dong and W. Horton, Phys. Fluids B 5, 1581 (1993).

9. W. Horton, J.Q. Dong, X.N. Su and T. Tajima, J. Geophys. Res. 98, 13377 (1993).

10. M. Artun and W.M. Tang, Phys. Fluids B 4, 1102 (1992).

11. F.L. Waelbroeck, T.M. Antonsen, Jr.,.P.N. Guzdar and A.B. Hassam, Phys. Fluids B 4, 2441 (1992).

12. S.P. Hirshman, Phys. Fluids 21, 224 (1978).

13. A.B. Hassam and R.M. Kulsrud, Phys. Fluids 21, 2271 (1978).

14. S.P. Hirshman, Nucl. Fusion 18, 917 (1978). 
15. A.A. Ware and J.C. Wiley, Phys. Fluids 24, 936 (1981).

16. S.P. Hirshman and D.J. Si

17. R.D. Hazeltine, Phys. Fluids 17, 961 (1974).

18. X.N. Su, W. Horton, and P.J. Morrison, Phys. Fluids B 4, 1238 (1992).

19. X.N. Su, Drift Wave Coherent Vortex Structures in Inhomogeneous Plasmas, Dissertation, The Univ, of Texas at Austin, (1992).

2 A.D. Beklemishev and W. Horton, Phys. iluids'B 4, 200 and 2176 (1992).

21. H. Sugama, A.D. Beklemishev, and W. Horton, Comments on Single-helicity versus Multi-helicity Simulations, Proceedings of U.S.-Japan Workshop on $\eta_{i}$ mode, Austin, Texas, January, 1993.

22. B.G. Hong and W. Horton, Phys. Fluids B 2, 978 (1990).

23. B.A. Carreras, K. Sidikman, P.H. Diamond, P.W. Terry, and L. Garcia, Phys. Fluids B 4, 3115 (1992).

24. A.B. Hassam, T.M. A tonsen, Jr., J.F. Drake, P.N. Guzdar, C.S. Liu, D.R. McCarthy, and F.L. Waelbroeck, Phys. Fluids B 3, 2519 (1993). 


\section{Figure Captions}

1. (a) Normalized growth rate contour in the $\left(u_{\|}^{\prime}, u_{1}^{\prime}\right)$ plane for magnetic shearless configuration with $k_{y} \rho_{s}=1$ and $S_{0}=0.2$. (b) Normalized growth rate contour in the ( $u_{\|}^{\prime}$, $\left.u_{\perp}^{\prime}\right)$ plane for slab geometry with magnetic shear, $k_{y}=1$ and $s=0.1$.

2. Time evolutions of (a) the turbulent fluctuation energy $E_{f l, 1}$ and (b) perpendicular background flow energy $E_{b g, 1}$ in case $1 \mathrm{~A}$. For $t<300\left(L_{n} / c_{s}\right)$, the perpendicular background flow $u_{\perp}=0$ fixed. When $t>300\left(L_{n} / c_{s}\right), u_{\perp}$ evolves with time according to Eq. (16) with $\nu^{n c}=0$ and $\nu_{\perp}^{a}=\nu_{\|}^{a}=0$.

3. Time evolutions of (a) the turbulent fluctuation energy $E_{f l, 2}$ and (b) parallel background flow energy $E_{b g, 2}$ in case 1A. For $t<300\left(L_{n} / c_{s}\right)$, the parallel background flow $u_{\|}=6 \sin \left(k_{1} x\right)\left(\rho_{s} c_{s} / L_{n}\right)$ fixed. When $t>300\left(L_{n} / c_{s}\right), u_{\|}$evolves with time according to Eq. (17) with $\nu^{n c}=0$ and $\nu_{\perp}^{a}=\nu_{\|}^{a}=0$.

4. The contour plot of $\varphi(x, y, t)$ in case $1 \mathrm{~A}$. (a) At $t=275\left(L_{n} / c_{s}\right)$ and (b) at $t=$ $395\left(L_{n} / c_{s}\right)$.

5. Time evolutions of (a) the turbulent fluctuation energy $E_{f l, 1}$ and (b) perpendicular background flow energy $E_{b g, 1}$ in case 1B.

6. Time evolutions of (a) the turbulent fluctuation energy $E_{f l, 2}$ and (b) parallel background flow energy $E_{b g, 2}$ in case 1B.

7. Time evolutions of individual mode fluctuation of $\tilde{\varphi}\left(k_{x}, k_{y}=1.0\right)$ for a spectrum of $k_{x} \rho$ s values.

8. Time evolutions of (a) the turbulent fluctuation energy $E_{f l, 1}$ and (b) perpendicular background flow energy $E_{b g, 1}$ when a counter parallel stream of beam with speed $u_{\|}^{a}=$ 
$10 \sin \left(k_{1} x\right)\left(\rho_{s} c_{s} / L_{n}\right)$ is being injected ir. he plasma in case 2.

9. Time evolutions of (a) the turbulent fluctuation energy $E_{f l, 2}$ and (b) parallel background flow energy $E_{b g, 2}$ when a counter parallel stream of beam with speed $u_{\|}^{a}=$ $10 \sin \left(k_{1} x\right)\left(\rho_{s} c_{s} / L_{n}\right)$ is being injected into the plasma in case 2.

10. Time evolution of the total fluctuation energy $E_{f l, 2}(t)$ in case 2 . The dashed line shows the $E_{f l}$ versus time $t$ and the solid line is the fitting curve obtained from the nonlinear regression fit to the parameterization $E_{f l, 2}(\infty)+\Delta E / t^{m}$.

11. .e evolutions of (a) the turbulent fluctuation energy $E_{f l, 1}$ an (b) perpendicular i...kground flow energy $E_{b g, 1}$ with neoclassical coefficient $\nu^{\text {nc }}=0.01$ in case 3 .

12. Time evolutions of (a) the turbulent fluctuation energy $E_{f l, 2}$ and (b) parallel background flow energy $E_{b g, 2}$ with neoclassical coefficient $\nu^{n c}=0.01$ in case 3 .

13. Time evolution of the quantity $E_{n c}=\left\langle\left(u_{\perp}-S_{0} u_{\|}+u^{n c}\right)^{2}\right\rangle$ in case 3 .

14. Qualitative description of the background flow state evolution. The curve represents the marginal instability of the parallel shear flow driven mode and the straight line represents the equilibrium path line $u_{\perp}-S_{0} u_{\|}+u^{n c}=0$ in case 3.

15. Time evolutions of the total fluctuation energy of a) fixed background ( $u_{\perp}$ and $u_{\|}=0$ ) and (b) evolving background (with $\nu^{n c}=0$ ) in $\eta_{i}$ mode case in the time interval $t=0$ $200\left(L_{n} / c_{s}\right) \cdot($ case 4$)$.

16. Time evolutions of (a) the total fluctuation energy and (b) the background flow energy (with $\nu^{n c}=0$ ) in the time interval $t=0-1000\left(L_{n} / c_{s}\right)$. The saturation is observed at around $t \sim 600\left(L_{n} / c_{s}\right)$. During the saturation the oscillations of the background and fluctuation energies look like mirror image of each other. 
17. Time evolutions of perpendicular background flow energies for neoclassical coefficients (a) $\nu^{n c}=0.01$ and (b) $\nu^{n c}=0.1$ in case 4 .

18. Time evolutions of the total fluctuation energy for neoclassical coefficients (a) $\nu^{\text {nc }}=$ 0.01 and (b) $\nu^{n c}=0.1$ in case 4.

19. Time evolutions of (a) the total fluctuation energies and (b) the total background energies for different neoclassical coefficients. Dot, solid and dashed lines are for $\nu^{n c}=0.1$, 0.01 and 0 , respectively. 


\begin{tabular}{|c|c|c|}
\hline$\nu^{n c}$ & $\sqrt{2 E_{f \ell, 1}}$ & $\sqrt{2 E_{b g, 1}}$ \\
\hline 0 & 50 & 50 \\
0.01 & 47 & 17 \\
0.1 & 45 & 7 \\
1.0 & 45 & 2.5 \\
\hline
\end{tabular}

Table I: Variation of Final Fluctuation and Background Energies with the Neoclassical Damping 


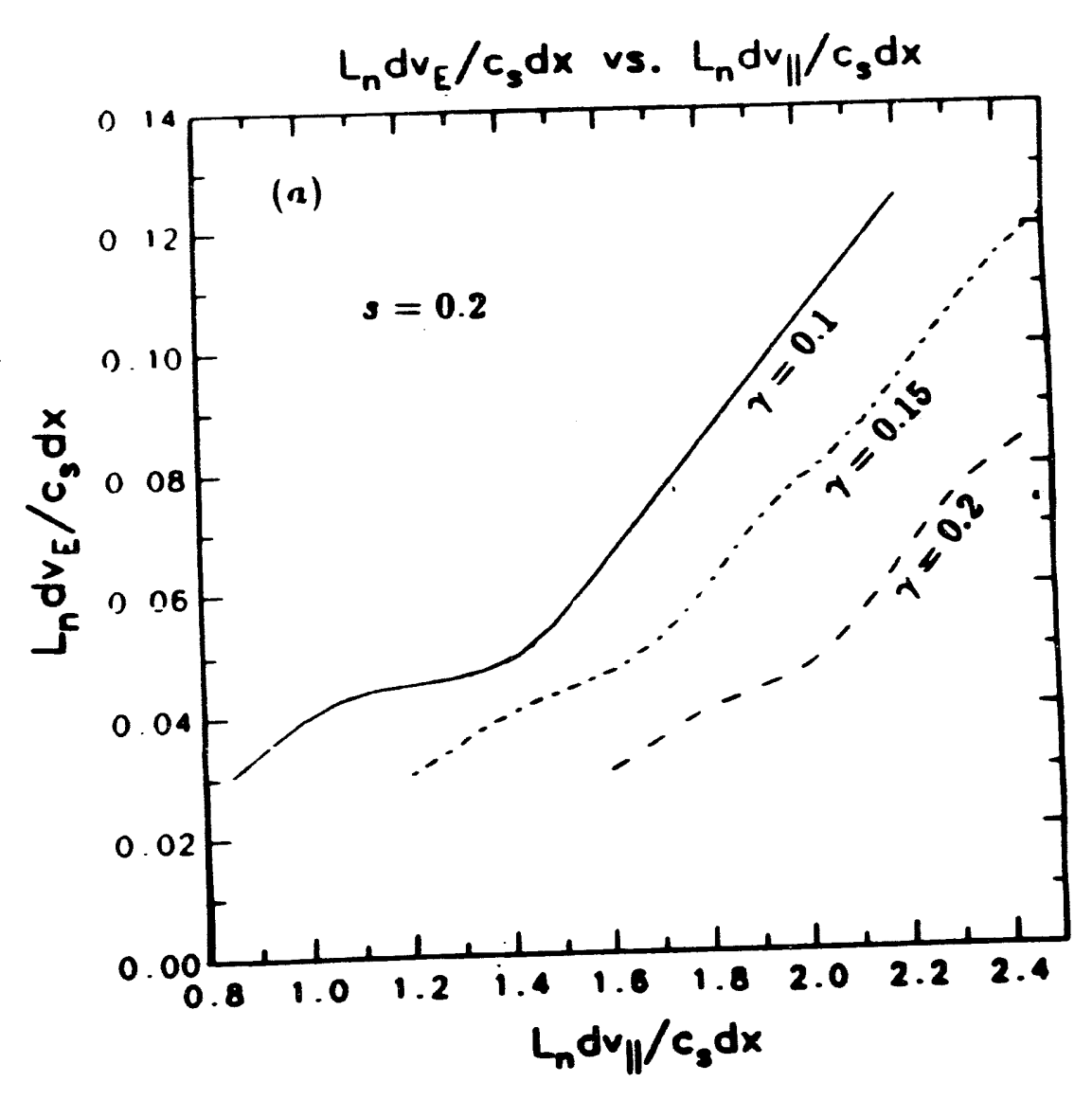

Fig. 1(a)

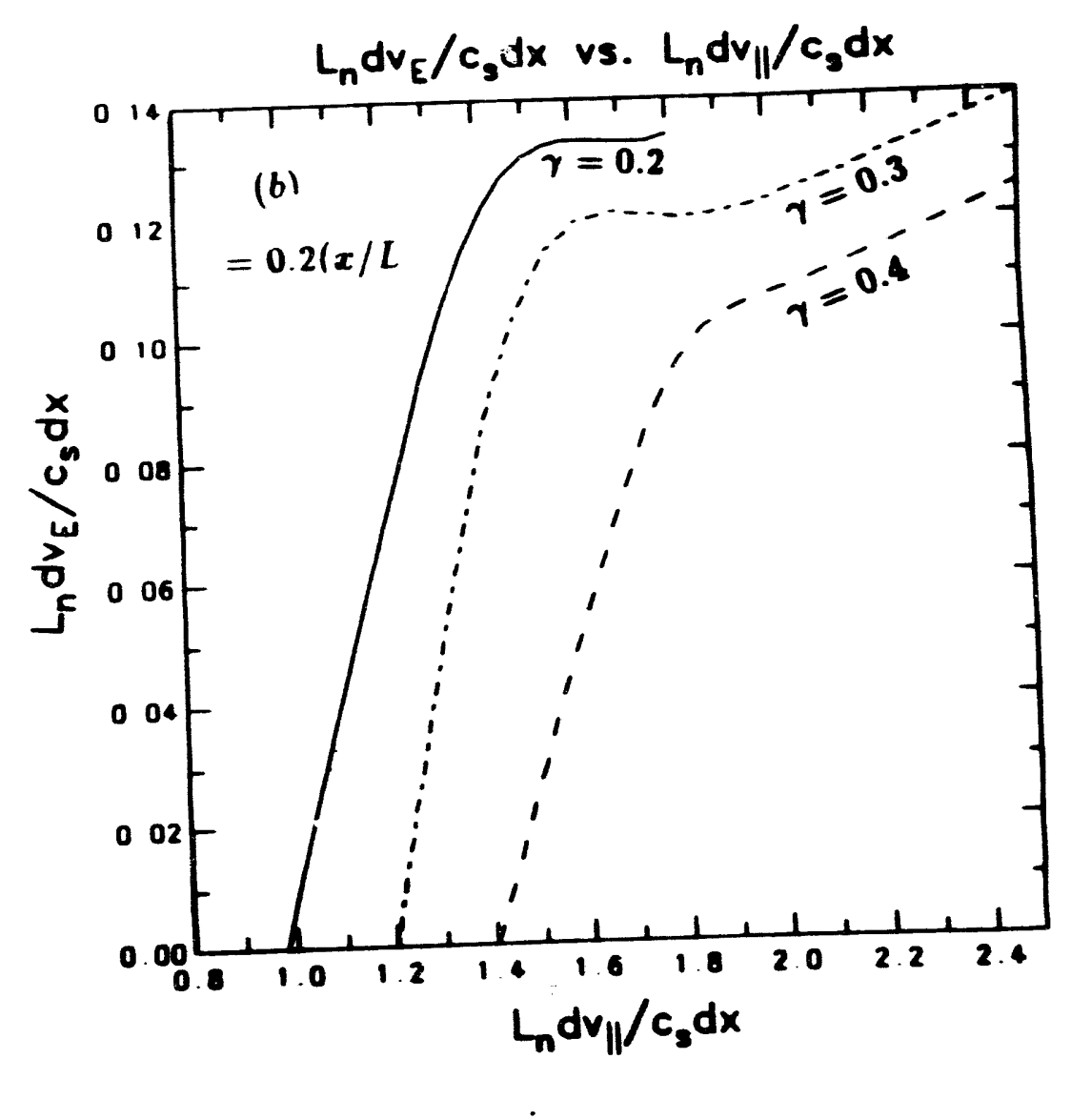

Fis. 1(b) 

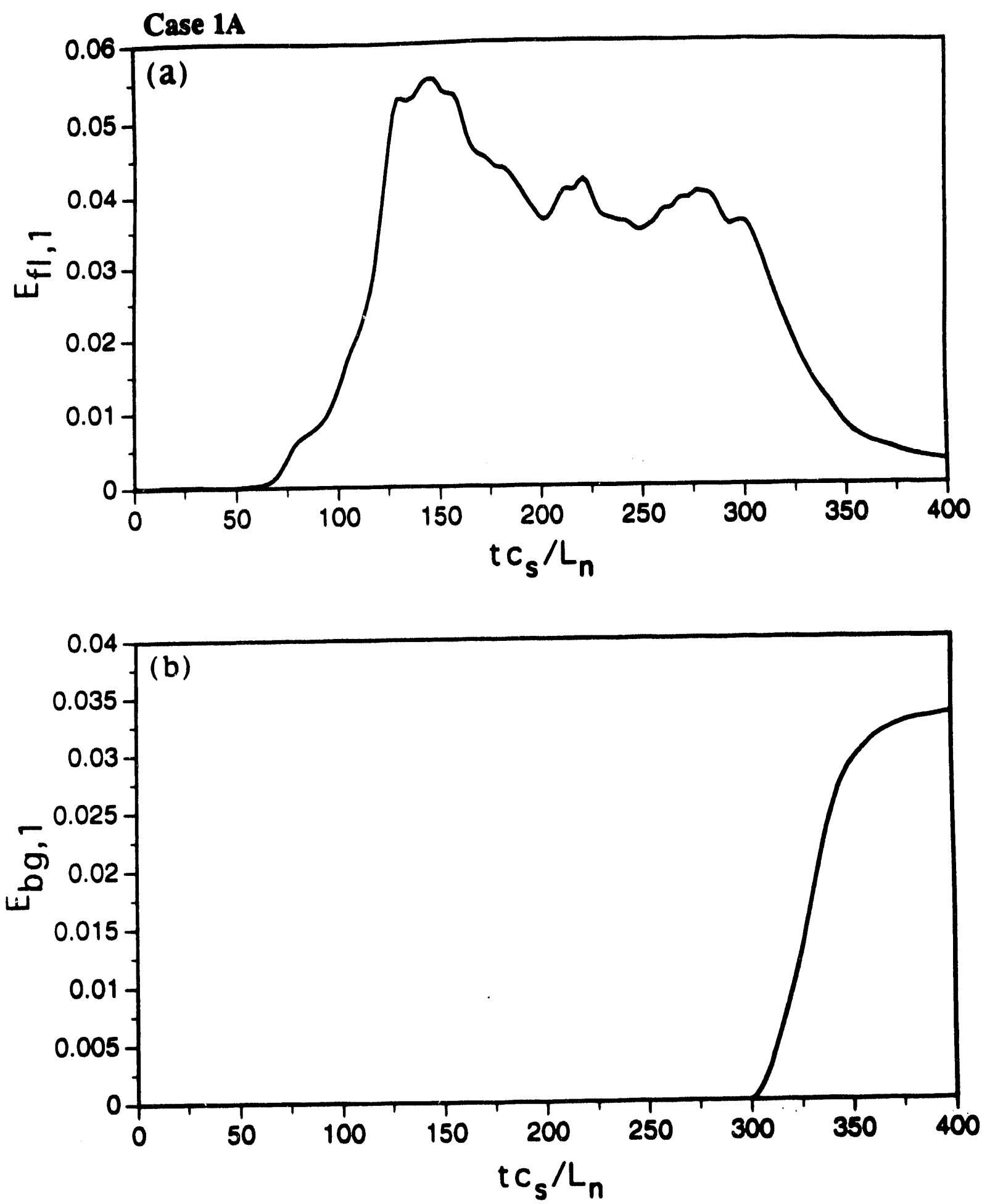

Figure 2 

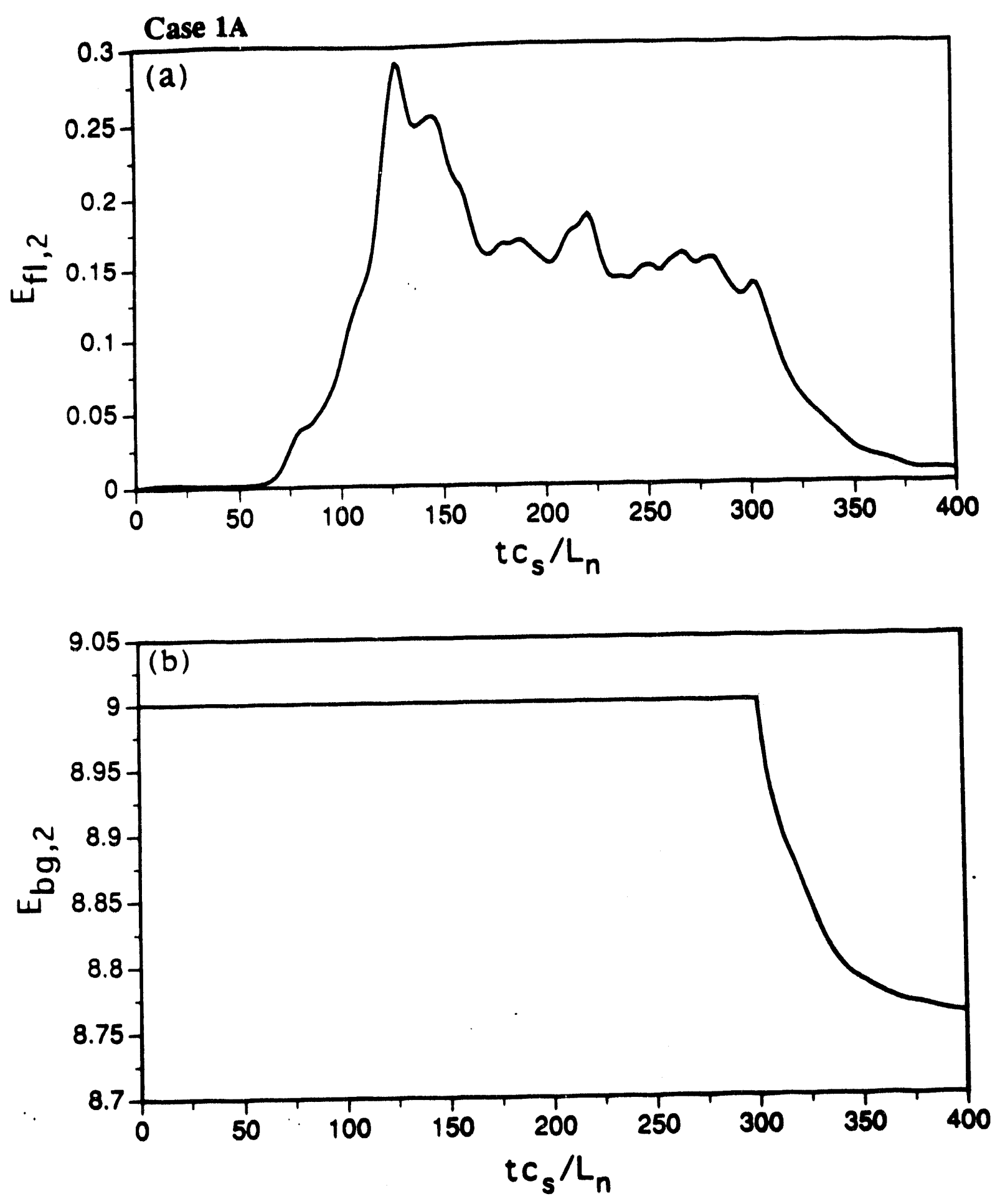

Figure 3 


\section{Case 1A}

(a) $r C_{s} / L_{n}=270$

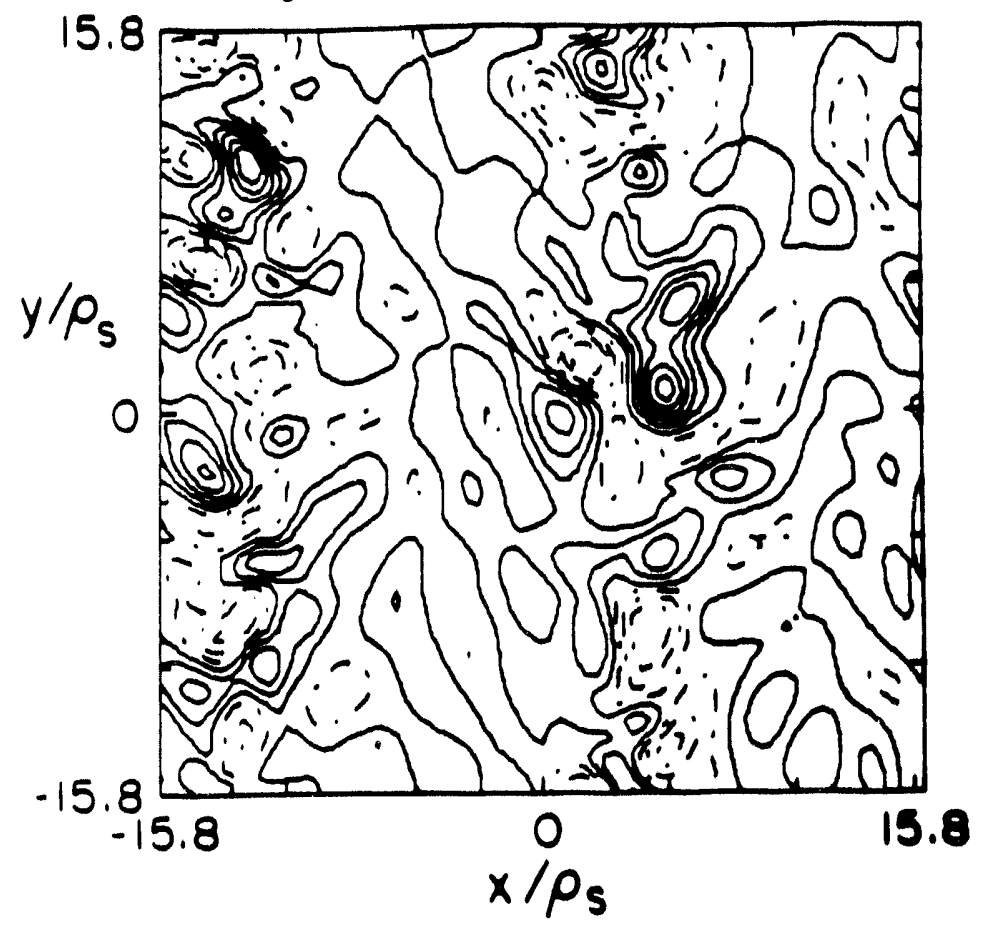

(b) $+c_{s} / L_{n}=390$

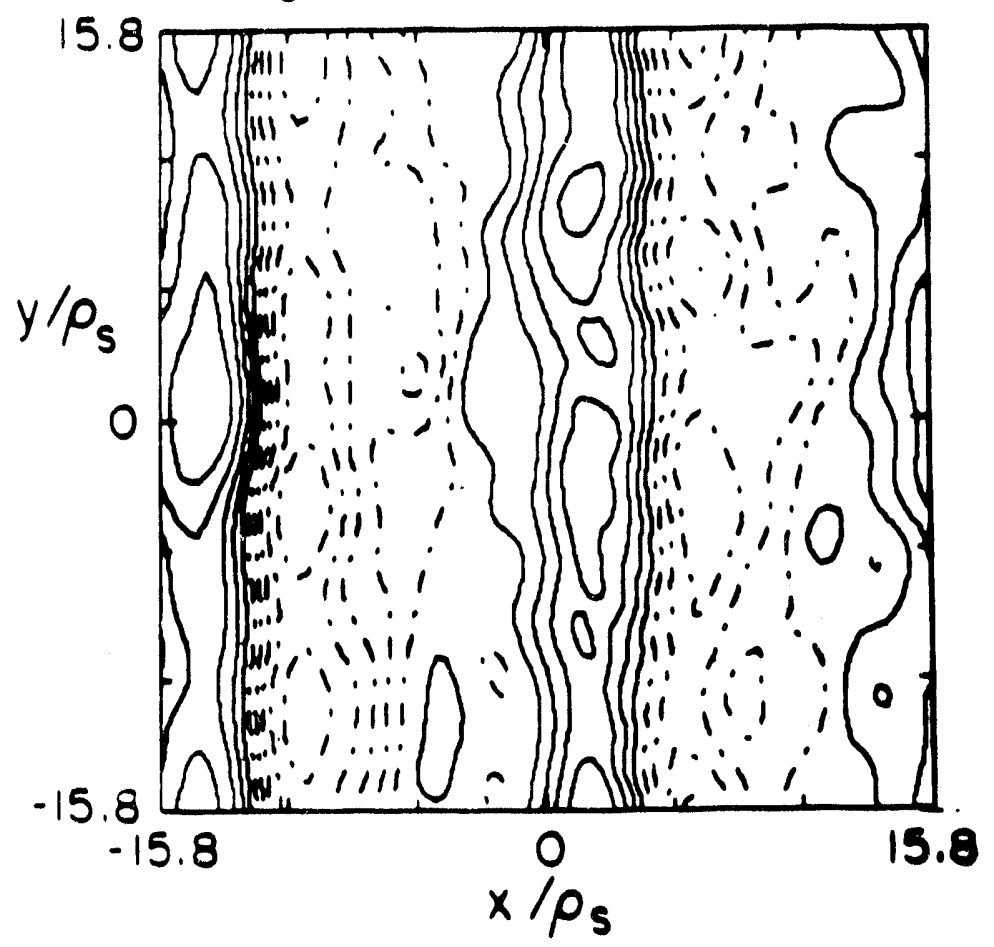

Figure 4 

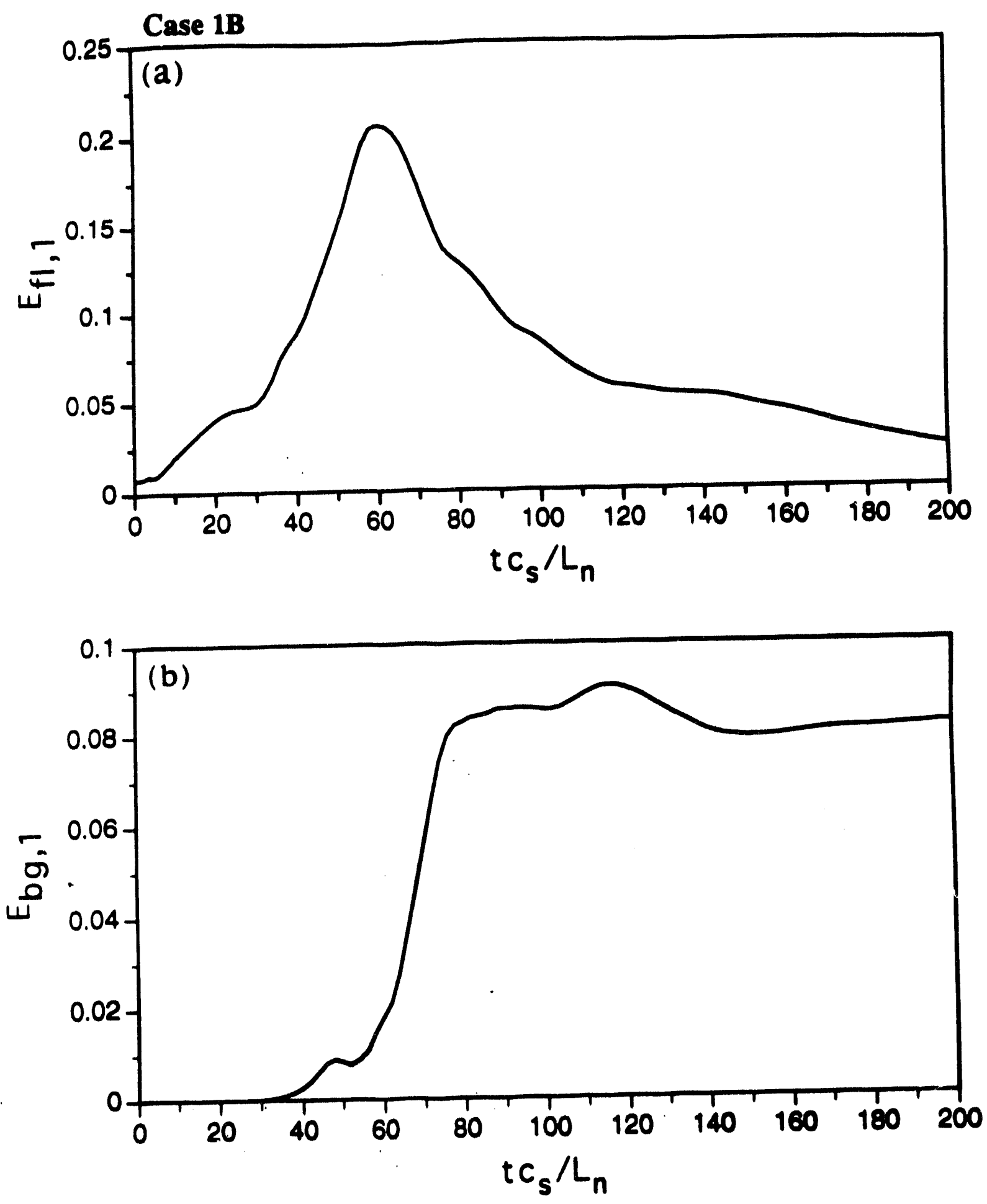

Figure 5 

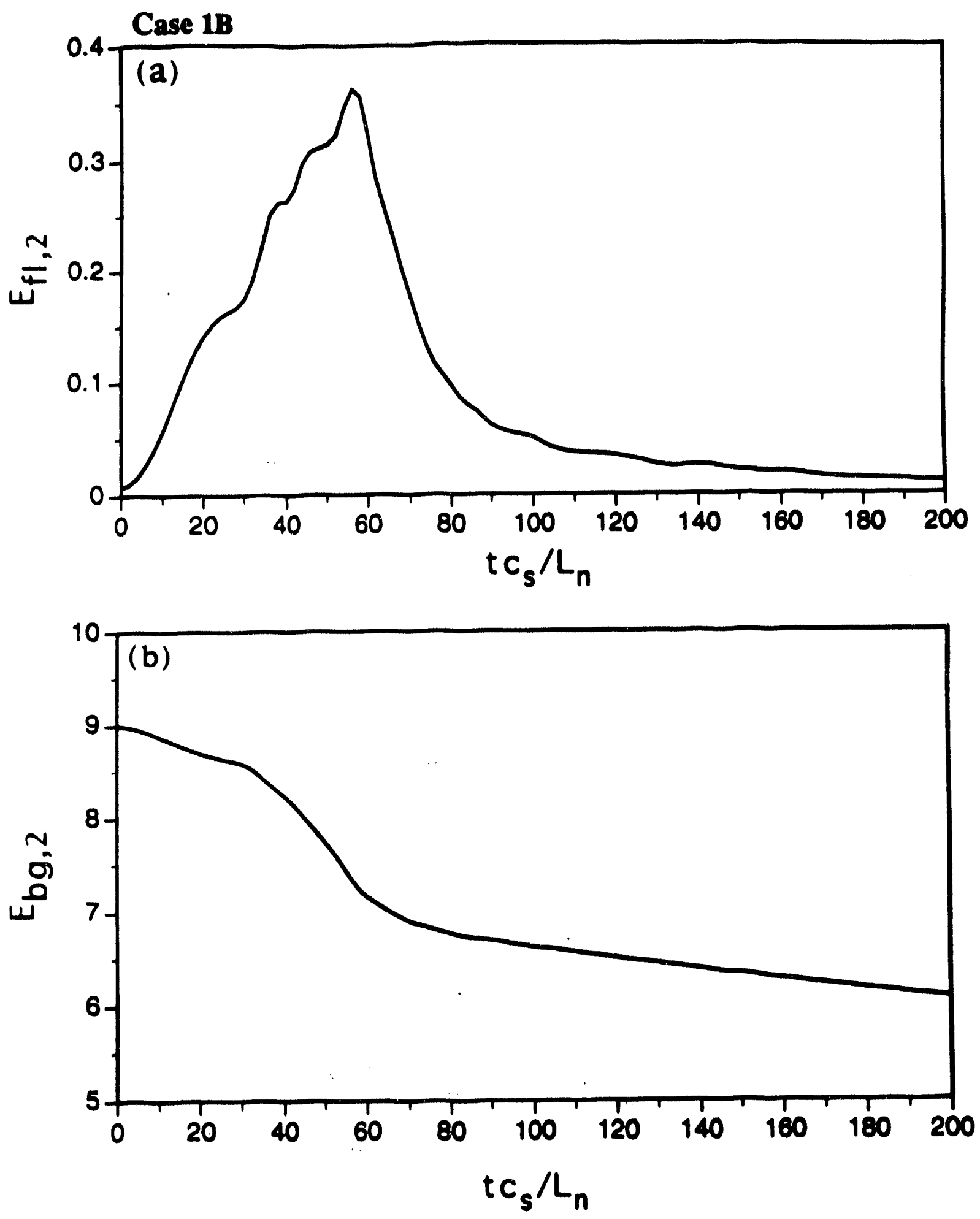

Figure 6 


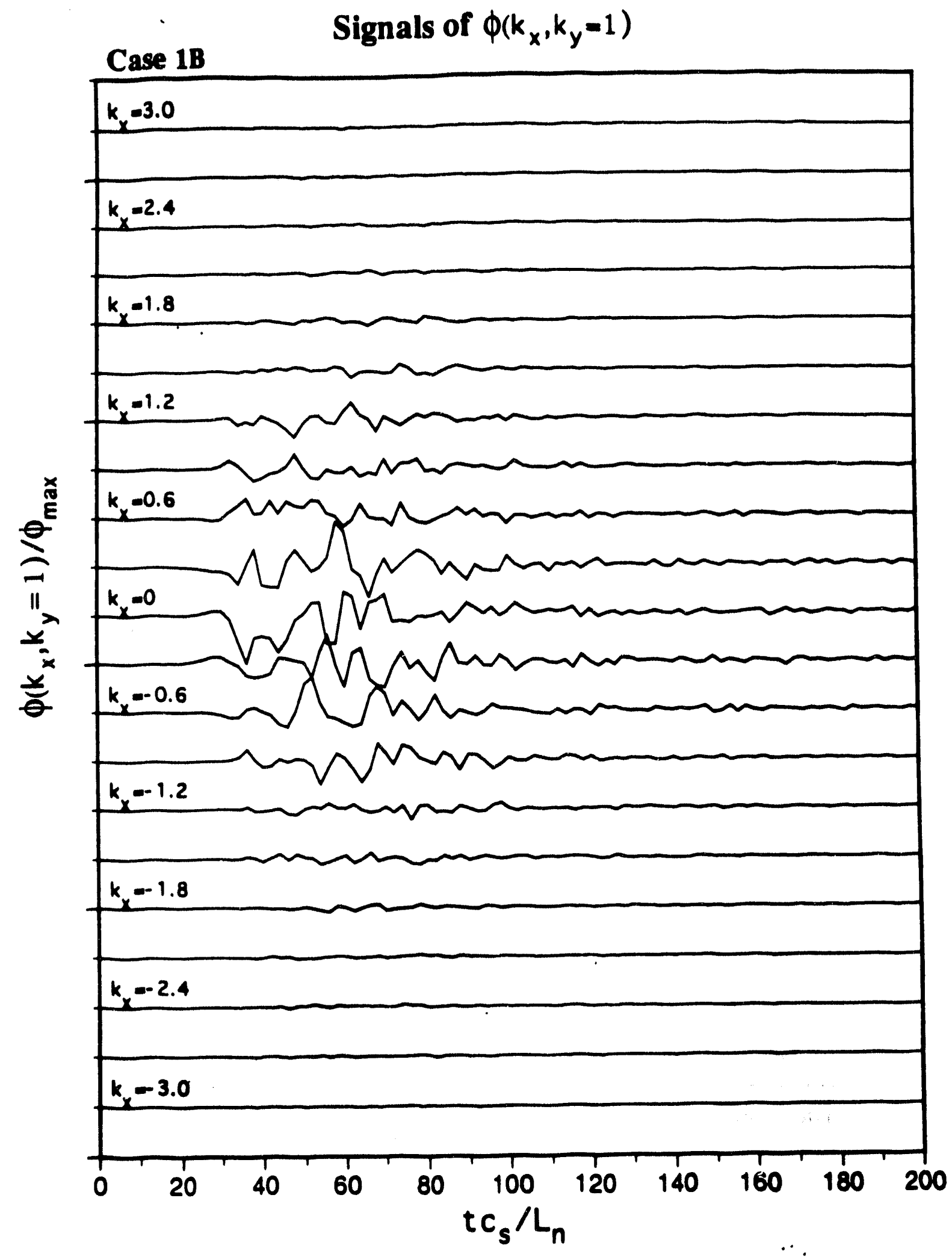

Figure 7 

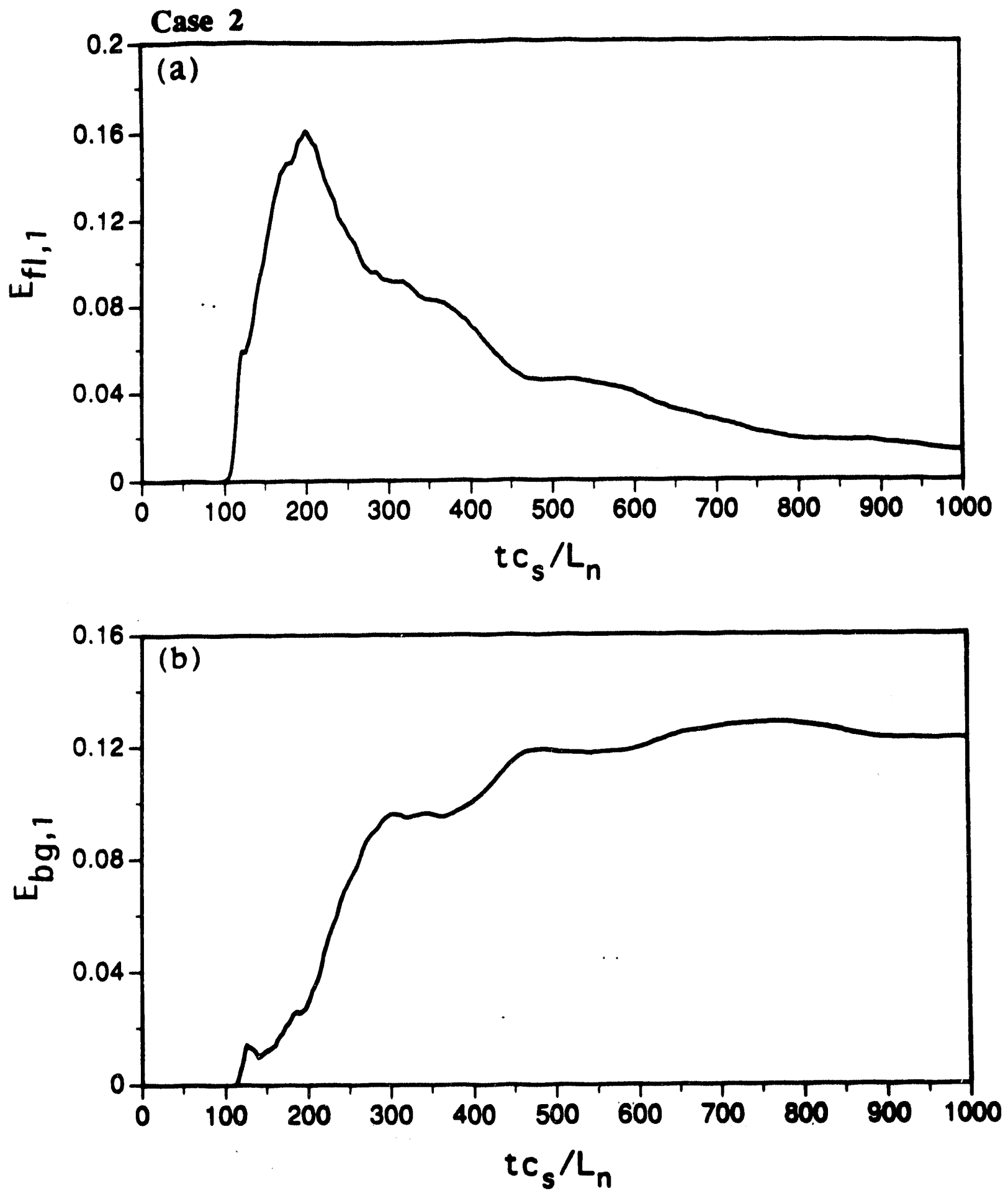

Figure 8 

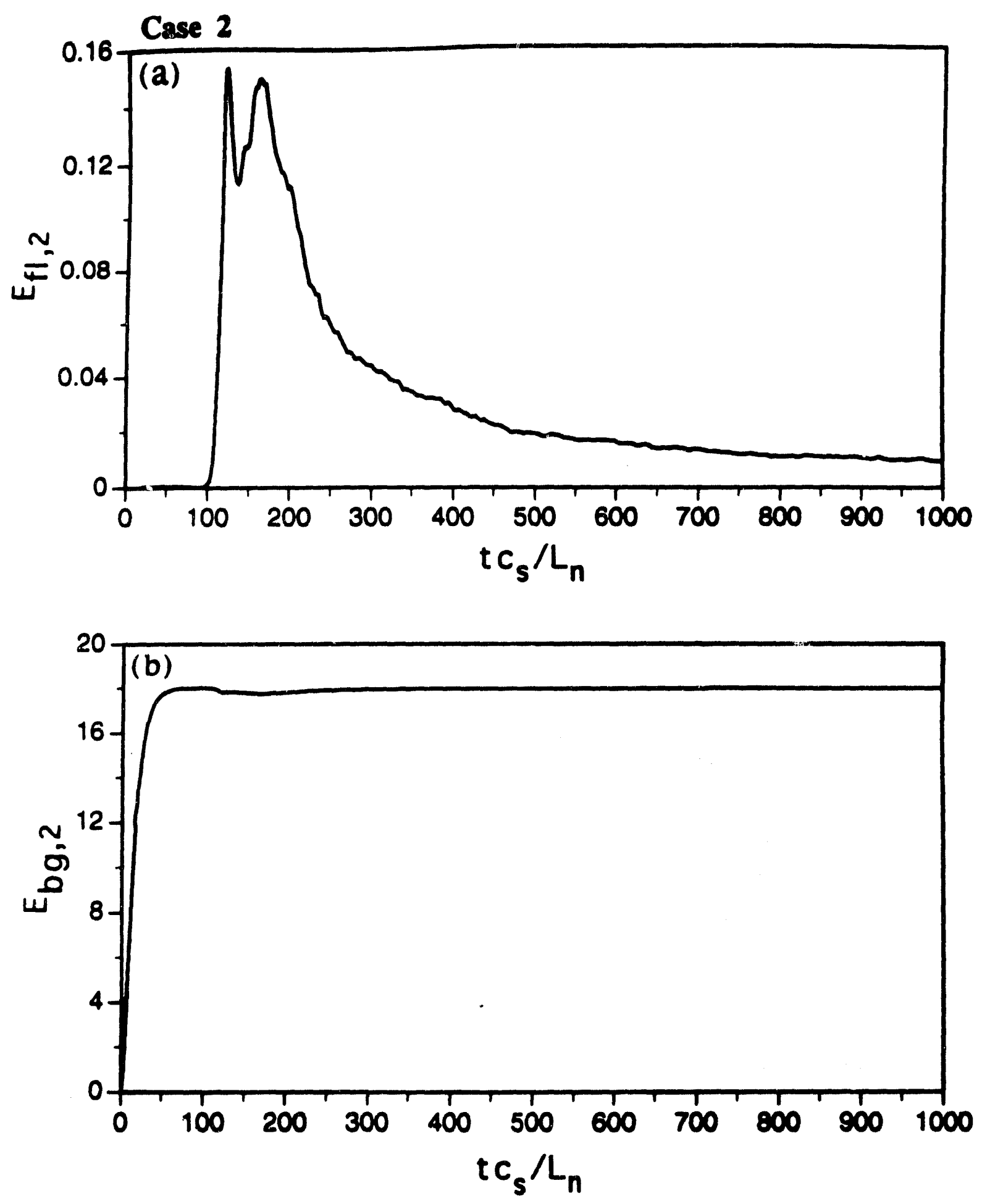

Figure 9 


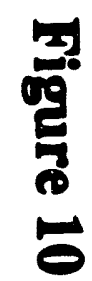

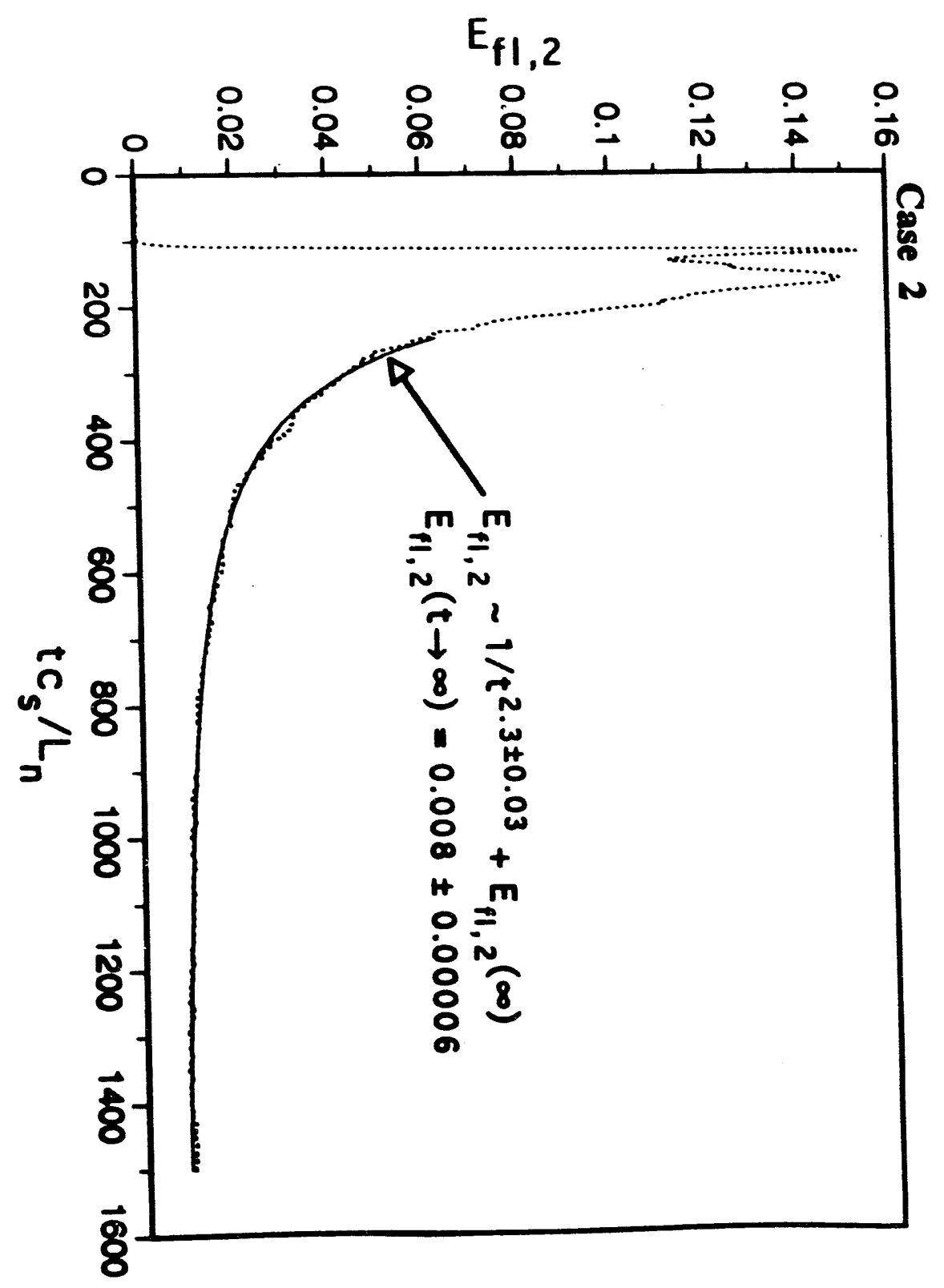



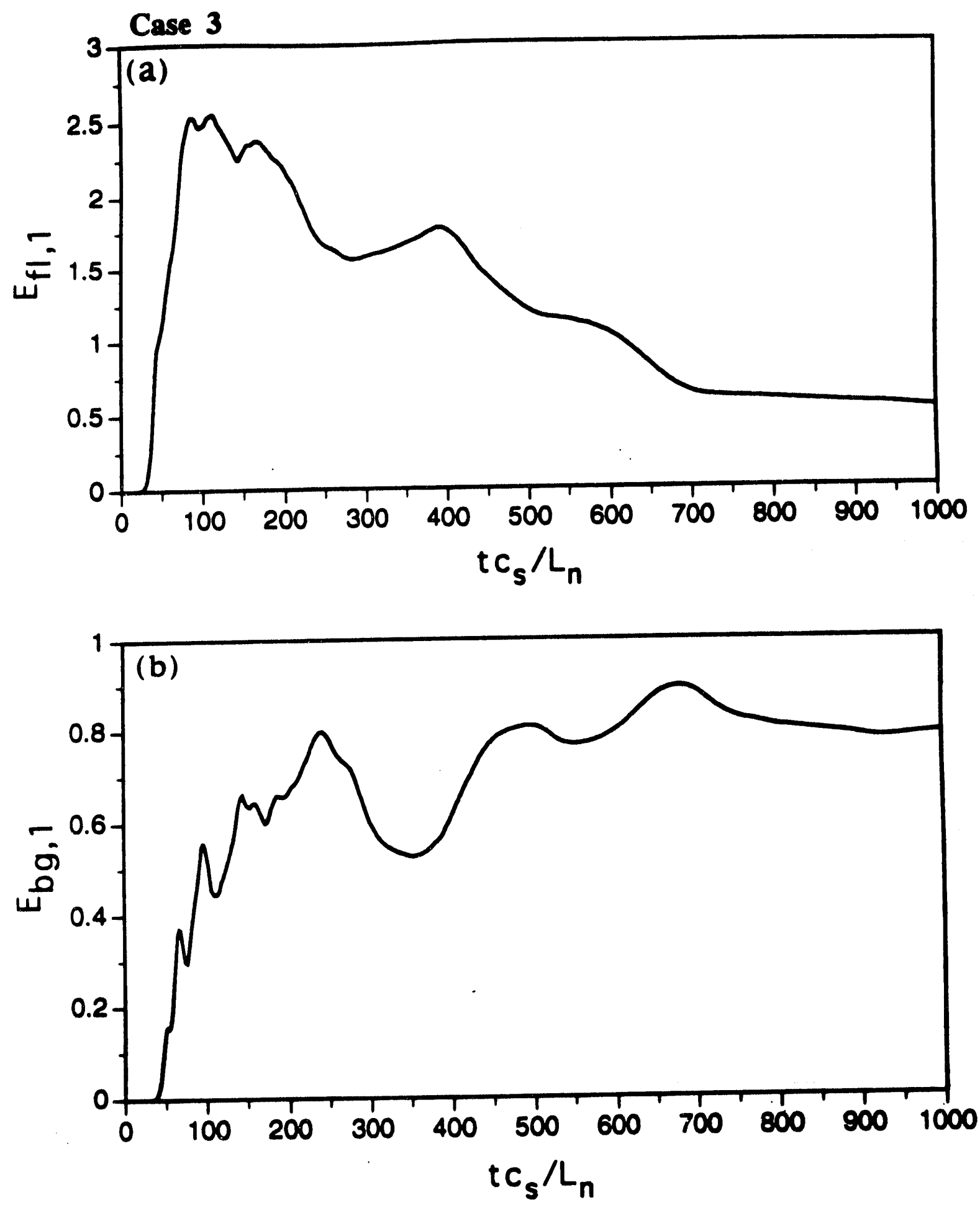

Figure 11 

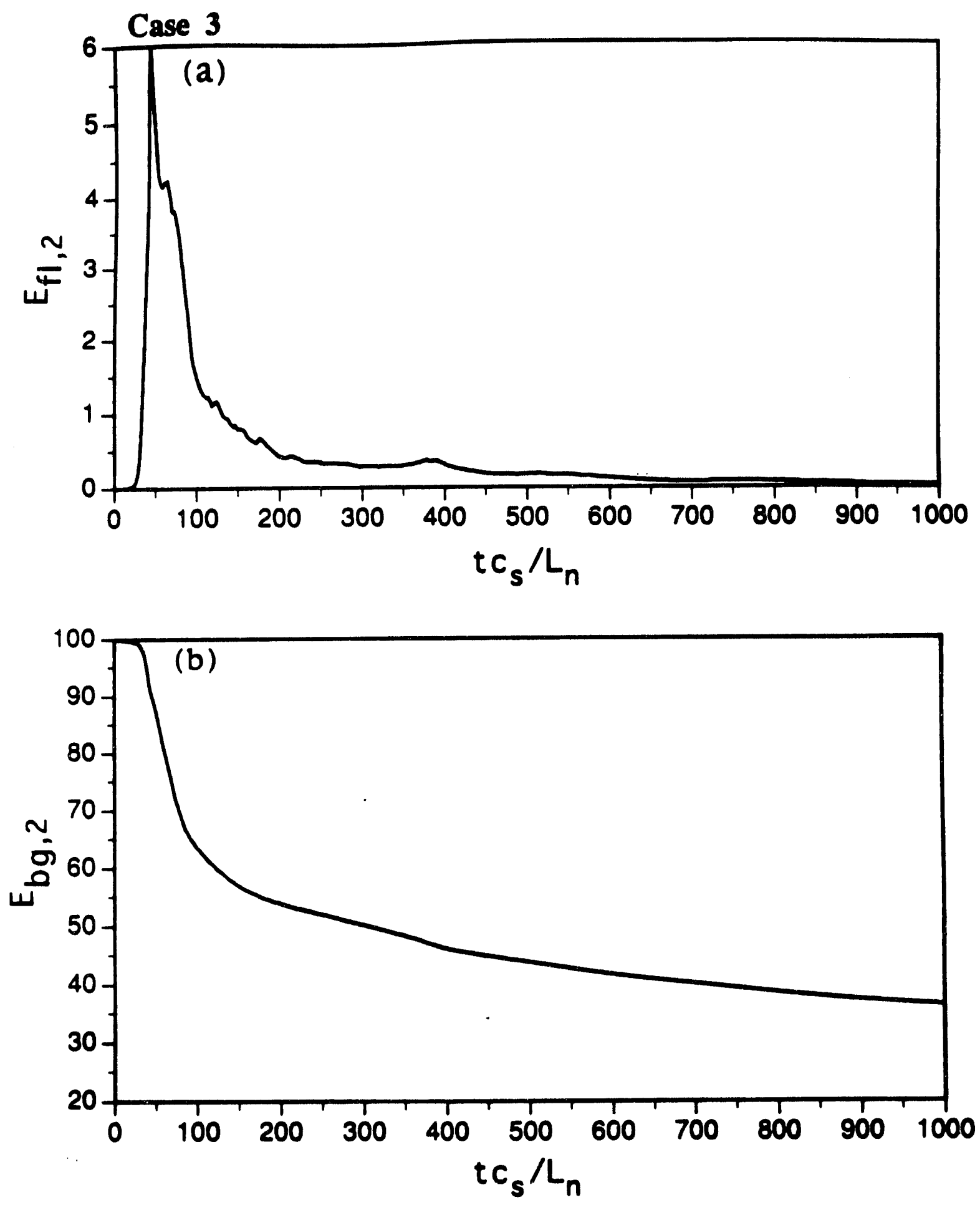

Figure 12 


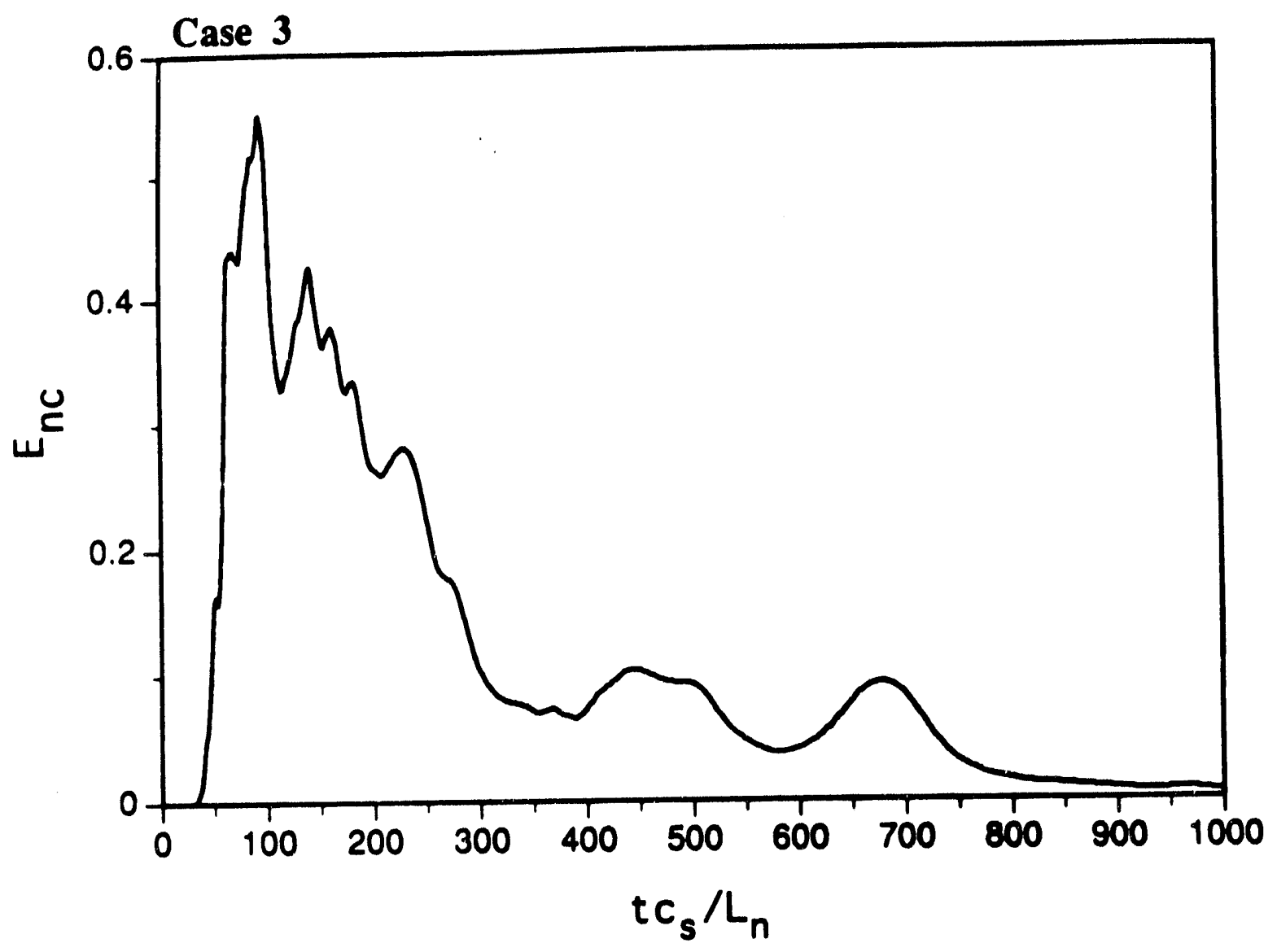

Figure 13 


\section{Case 3}

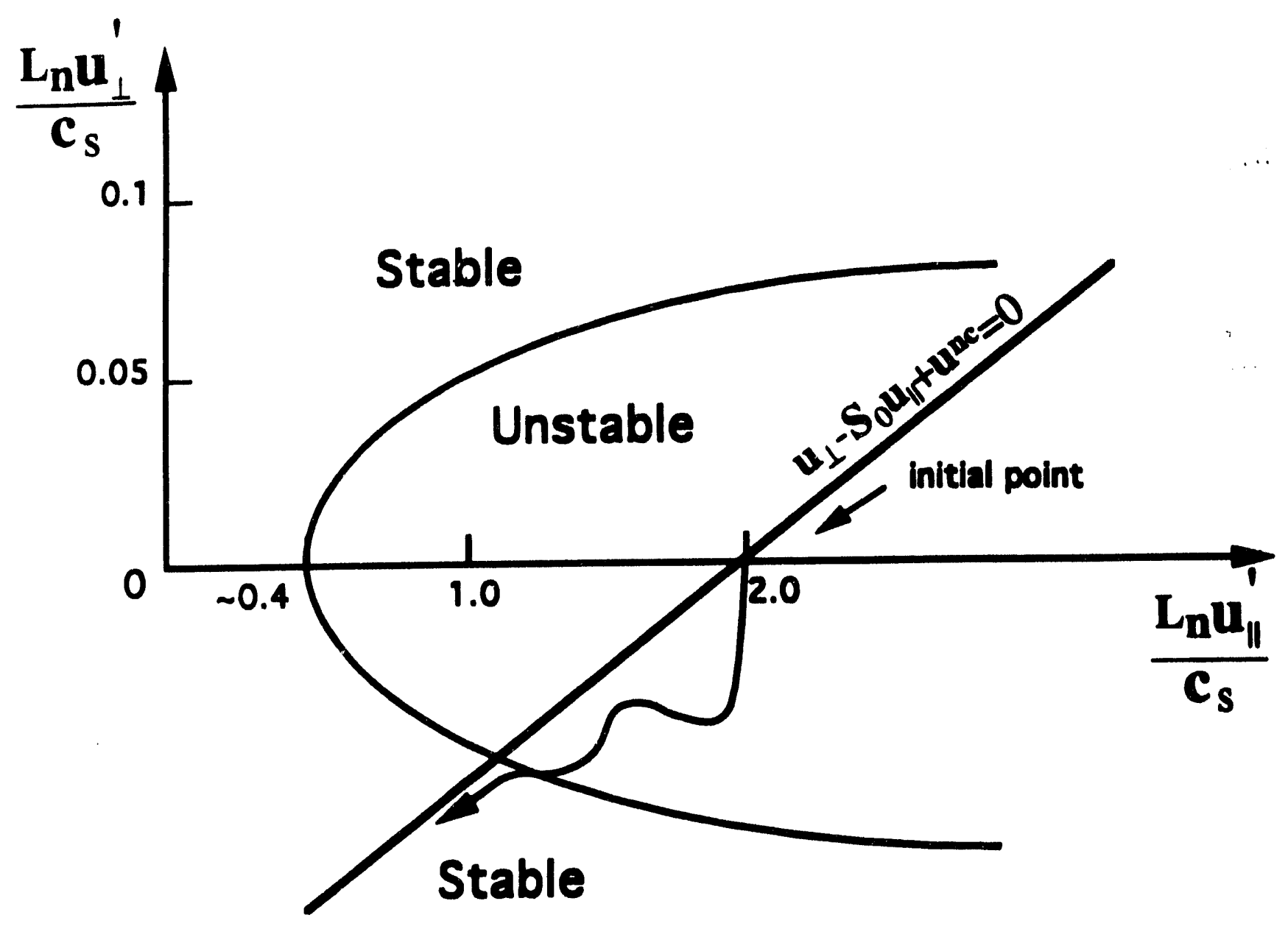

Figure 14 

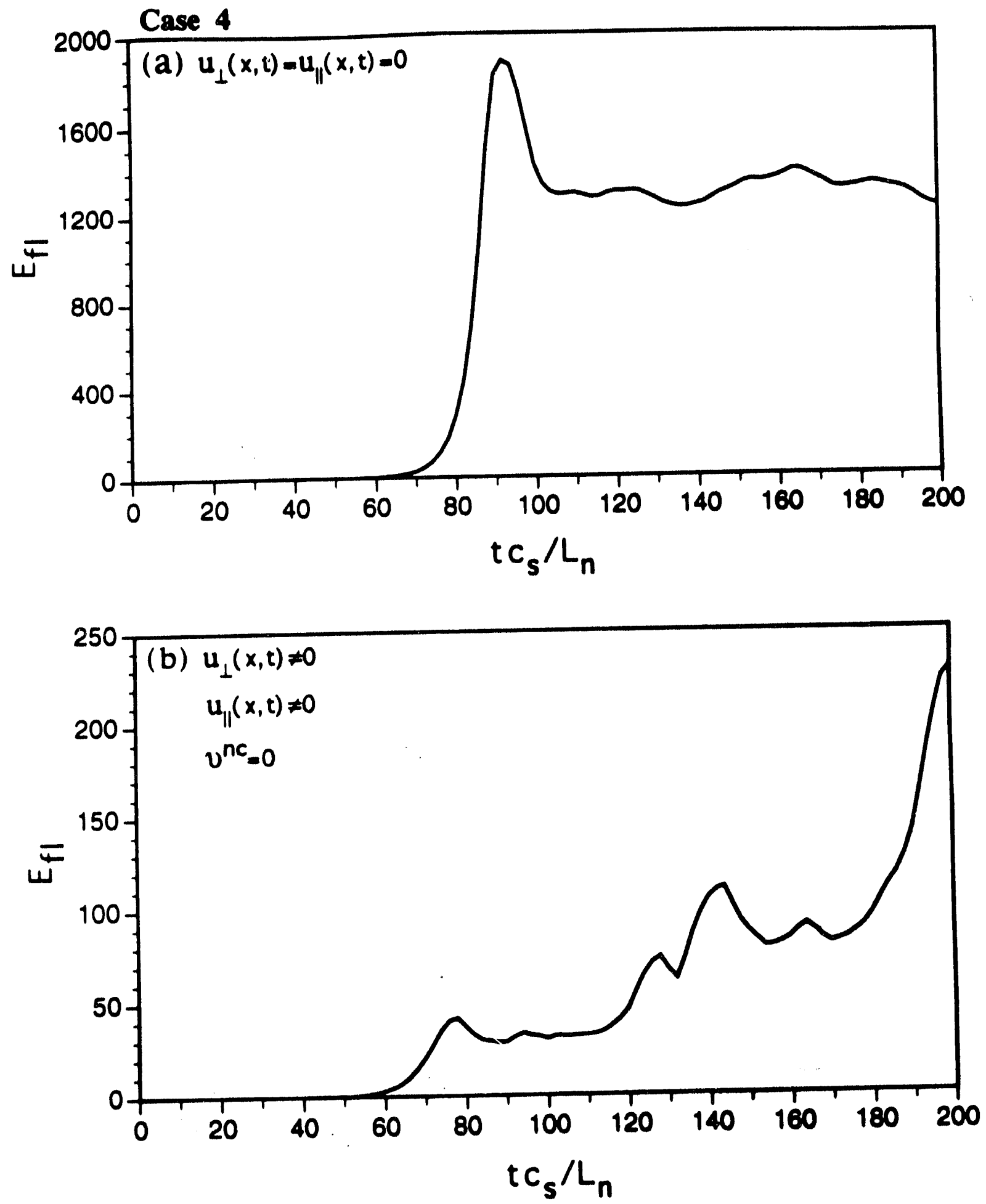

Figure 15 

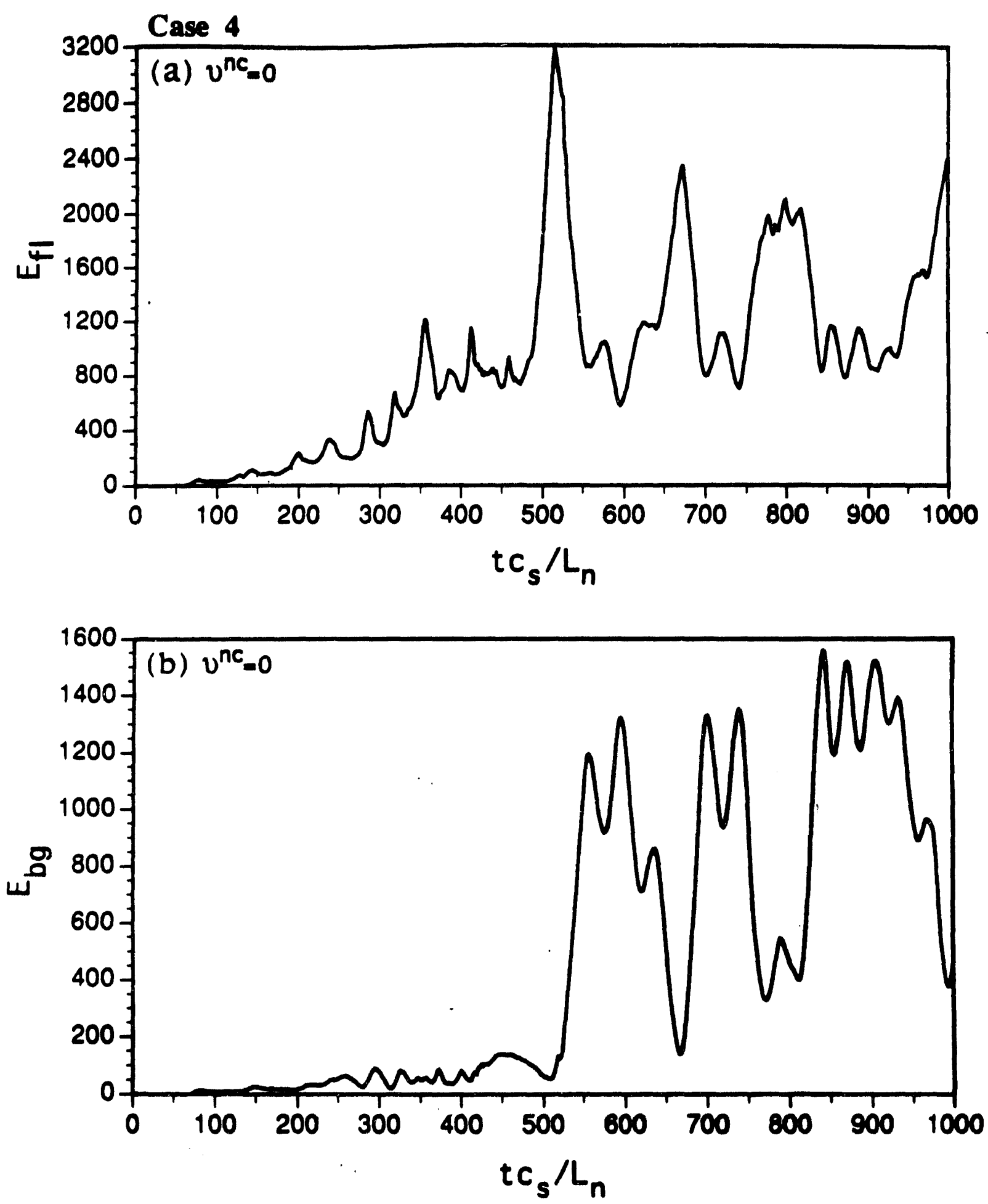

Figure 16 

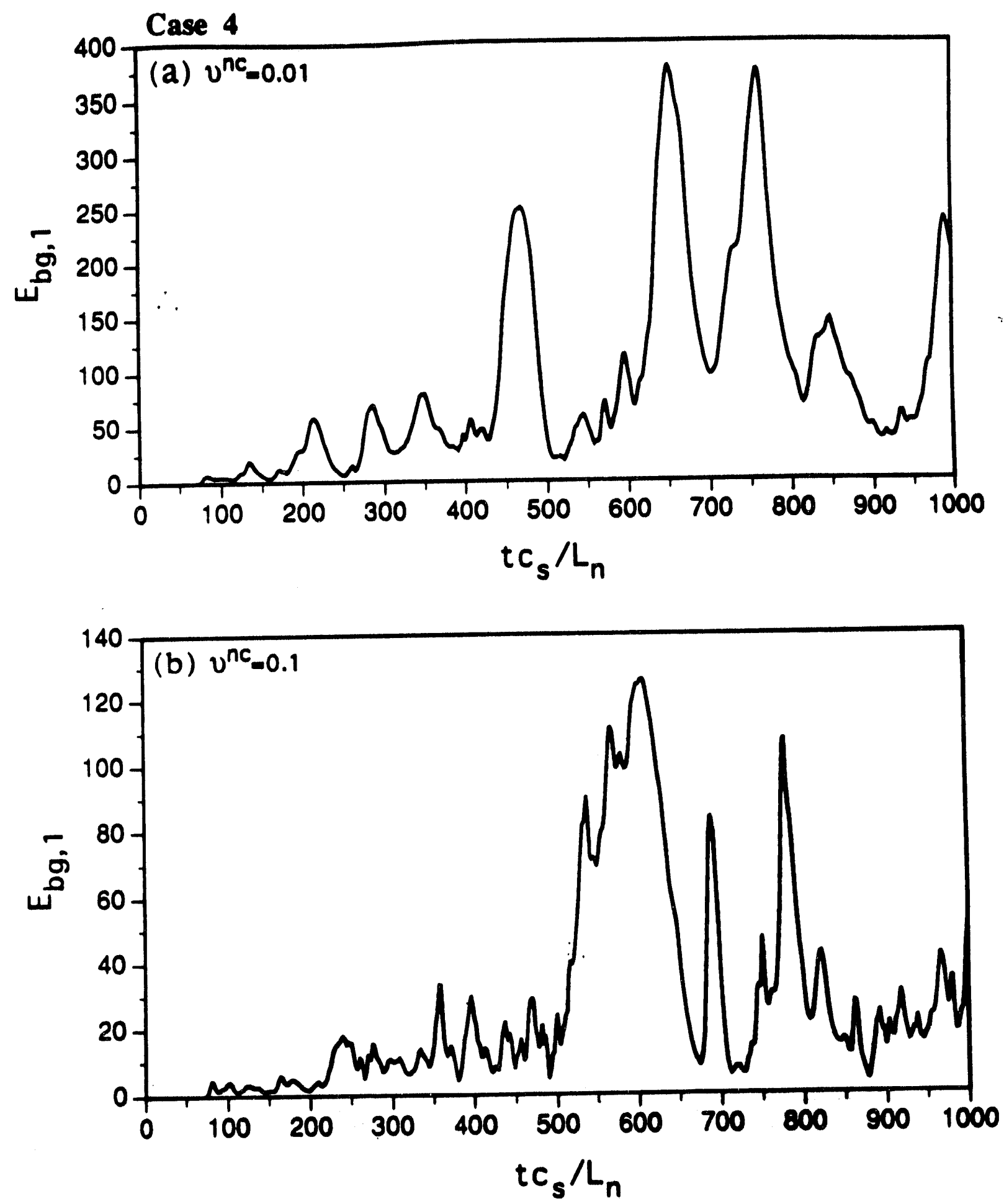

Figure 17 

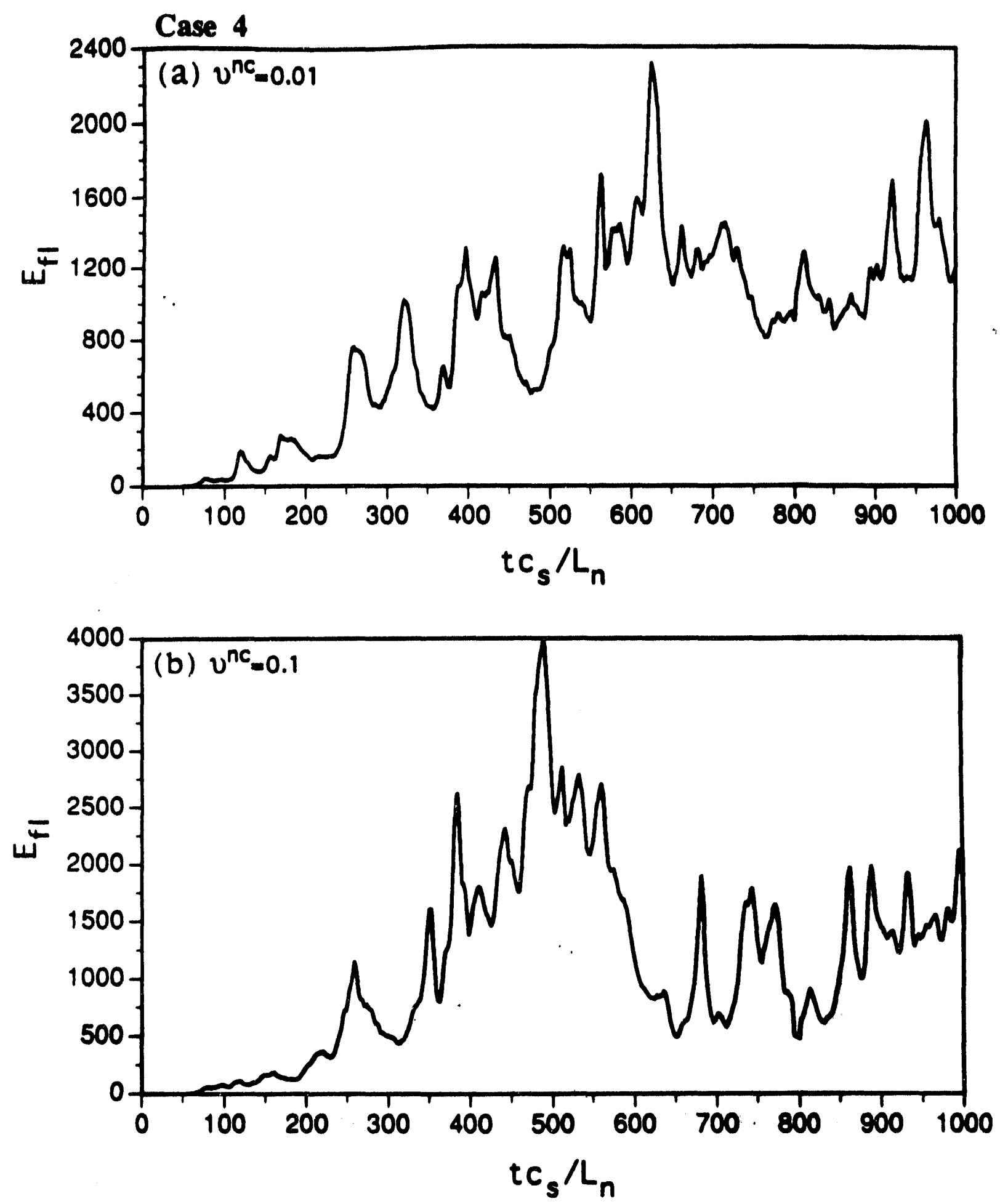

Figure 18 

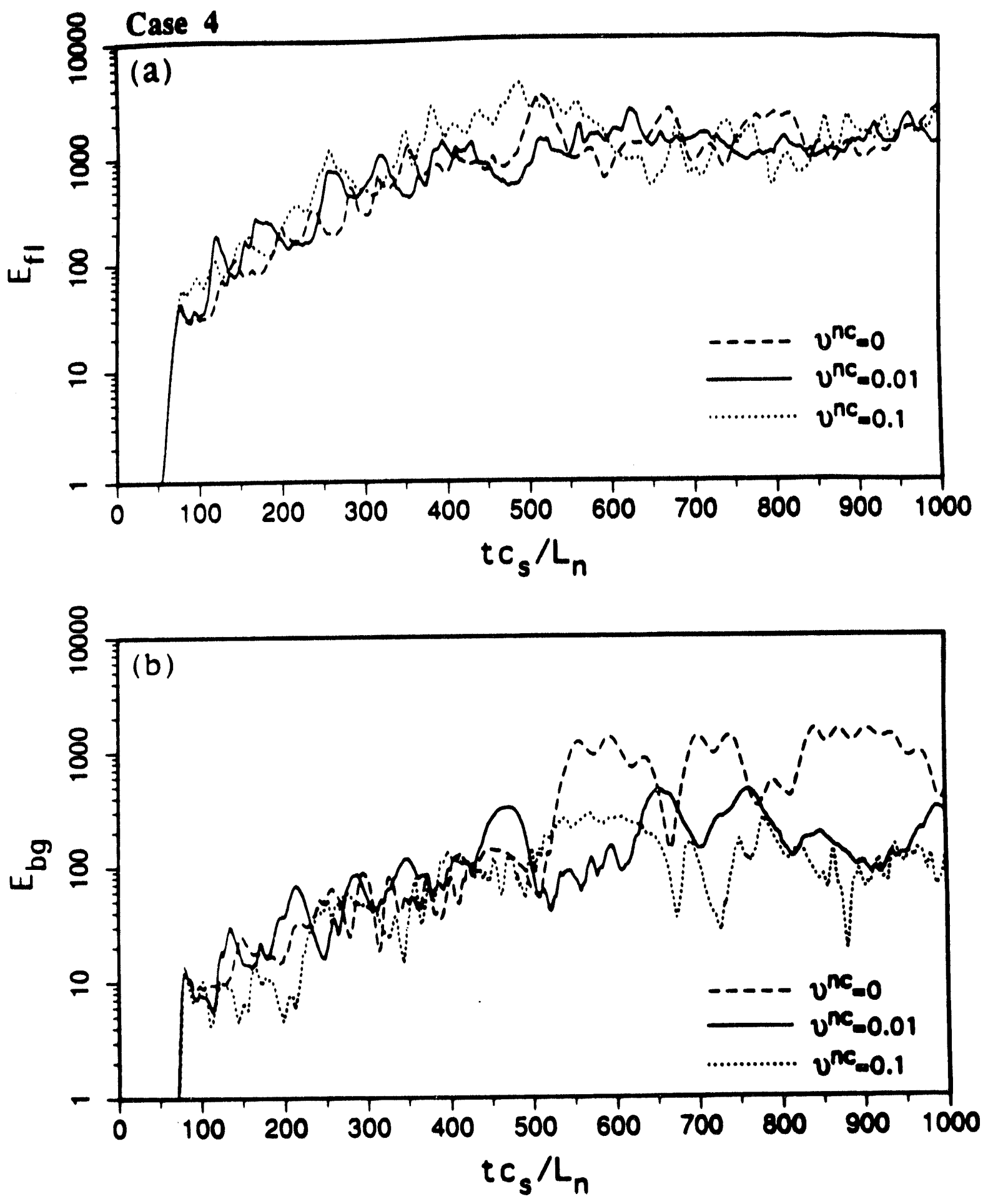

Figure 19 

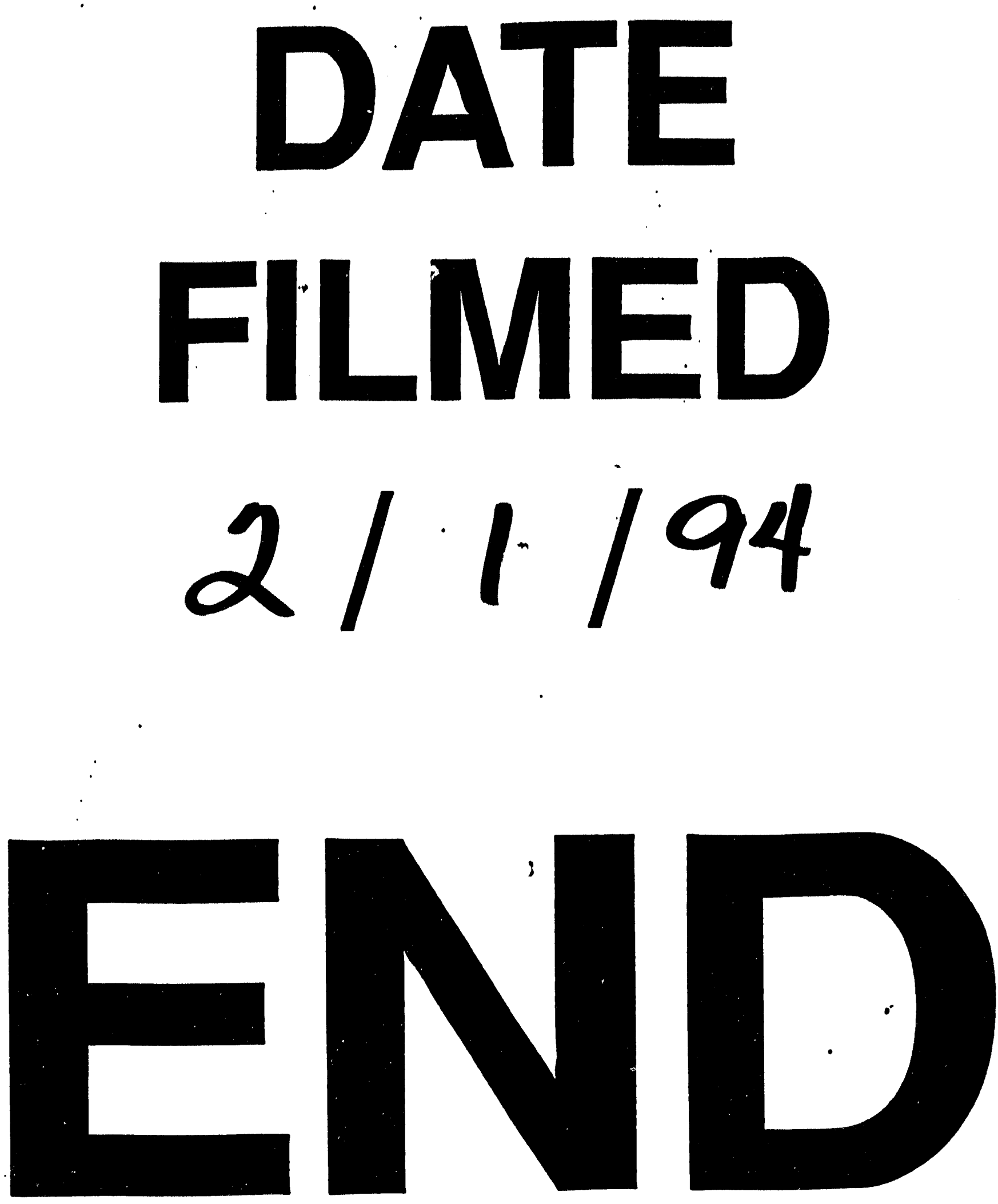
